\title{
Demersal assemblages of the continental shelf and upper slope of Angola
}

\author{
G. Bianchi \\ Institute of Marine Research, Division for International Development Programmes, PO Box 1870, Nordnes, \\ N-5024 Bergen, Norway
}

\begin{abstract}
The structure of the demersal assemblages (fish, crustaceans and cephalopods) of the continental shelf and upper slope of Angola (ca 5 to $17^{\circ} \mathrm{S}$ ) was studied based on the trawl survey of the RV 'Dr. Fridtjof Nansen' in February and March 1989, by means of an ordination technique, Detrended Correspondence Analysis (DCA), implemented by the computer program DECORANA and a classification technique, Two-Way Indicator species Analysis (TWIA), implemented by the computer program TWINSPAN. Correlation of DCA axes with the environmental variables showed that the thermal, depth-dependent stratification explains the main groupings, while bottom type and latitudinal gradients are the main factors within each depth stratum. A major latitudinal faunal shift takes place in the area Tombua-Cunene (Angola) and is related to the southern limit of Equatorial Water for the shallow-water assemblages and to the frontal area between the warm, southward-flowing Angola Current and the northward-flowing cold Benguela Current for the subthermocline shelf assemblages. Highest biomass densities (from bottom trawl catches) were found in correspondence with the upper slope, consisting mainly of the bony fish Synagrops microlepis (Norman).
\end{abstract}

\section{INTRODUCTION}

Within the framework of projects sponsored by UNDP/FAO and NORAD, the Norwegian RV 'Dr. F. Nansen' carried out acoustic and bottom trawl surveys on the Angolan shelf and upper slope in 1989. The present study, based on data collected through 1 bottom-trawl survey, has as a main objective to describe the different species assemblages in relation to the environmental variables and describe the general trends in the distribution of the bottom megafauna. Groups included in the analysis are bony fishes, elasmobranchs, stomatopods, decapod crustaceans and cephalopods.

The study of Angolan marine fish fauna is also of special interest because of the major changes in species composition taking place along its shelf. The latter extends from about 5 to $17^{\circ} \mathrm{S}$ and encompasses a typical tropical regime in its northern part as well as a temperate one, in the south, separated by the Benguela-Angola frontal system. It has indeed been recognized by several authors that a major zoogeographic boundary is present along the Angolan coast, separating the tropical fauna of Guinean origin from the temperate fauna associated with the Benguela system (Longhurst 1962). Da Franca (1968), however, points out that there is no really sharp boundary between 2 different faunal complexes. Faunas originating outside the Angolan coast meet and partially overlap along the Angolan shelf which should thus be considered as an area of biogeographic transition between the Guineo-equatorial province and the South African province. The present study, besides describing the main species assemblages found on the Angolan shelf, will also try to define more accurately the faunal transition area referred to above by more closely correlating the environmental parameters with the faunal patterns.

Several studies of the demersal communities on the continental shelf and upper slope off West Africa are available based on multivariate analysis techniques but none has covered Angola. Domain (1972) analyzed the assemblages of the Senegal-Gambia continental shelf using Principal Component Analysis (PCA) and later extended the study to Mauritania (Domain 1980) by cluster analysis and Correspondence Analysis; 
Fager \& Longhurst (1968) analyzed the demersal fish assemblages in the Gulf of Guinea based on the data from the Guinean Trawling Survey (GTS) with the multivariate analytical method described in Fager (1957); Lleonart \& Roel (1984) investigated the epibenthic fish and crustacean assemblages off Namibia, from 100 to $500 \mathrm{~m}$ depth, by means of hierarchical classification method, based on data collected through the Benguela II cruise in 1980; Mas-Riera et al. (1990) analysed the influence of the Benguela upwelling on the structure of the demersal fish populations of southern Namibia; Roel (1987) described the demersal communities off the west coast of South Africa by Correspondence Analysis.

\section{STUDY AREA}

Bottom topography and structure. The study area (Fig. 1) includes a coastline of ca 800 nautical miles (excluding Zaire), from about 5 to $17^{\circ} \mathrm{S}$ and covers trawlable grounds of the shelf and upper slope to ca $750 \mathrm{~m}$ depth.

Fig. 2 shows a map of the Angola shelf bottom based on analysis of the echograms, while Fig. 3 shows the position of bottom samples and type of sediment. The northern part of the area, to Pta. das Palmeirinhas, is characterized by large areas of fine to coarse sand. Silt is found outside the Congo River estuary, south of Cabinda, and north of Luanda. These areas are interrupted by beds of stones, rocks and corals (Fig. 2). The central part of the Angolan shelf, from south of Pta. das Palmeirinhas to Benguela, is also characterized by alternating fields of mud and fine to coarse sand, but silt and clay dominate large areas, and rocky bottoms are found mainly north of Cabo Ledo and off Cabeça da Baleia. The shelf between Tombua and the Cunene River estuary has a level bottom, with clay and silt in Baia dos Tigres and fine to coarse sand northwards to Tombua. The bottom is rough and untrawlable south of Baia dos Tigres, deeper than 100 to $200 \mathrm{~m}$.

Hydrology and biological oceanography. The general climatology of the Gulf of Guinea has been described by Wauthy (1983), including the Canary Current and Benguela Current frontal systems delimiting the tropical region north and south of the Equator respectively. The physical oceanography off Southern Angola has been described by Dias (1983) and features of the frontal system by Shannon et al. (1987). The survey report by Strømme \& Sæetersdal (1991) gives a description of the oceanographic conditions off Angola. The productive systems of the eastern tropical Atlantic between $20^{\circ} \mathrm{N}$ and $15^{\circ} \mathrm{S}$ were described and compared by Voituriez \& Herbland (1982).
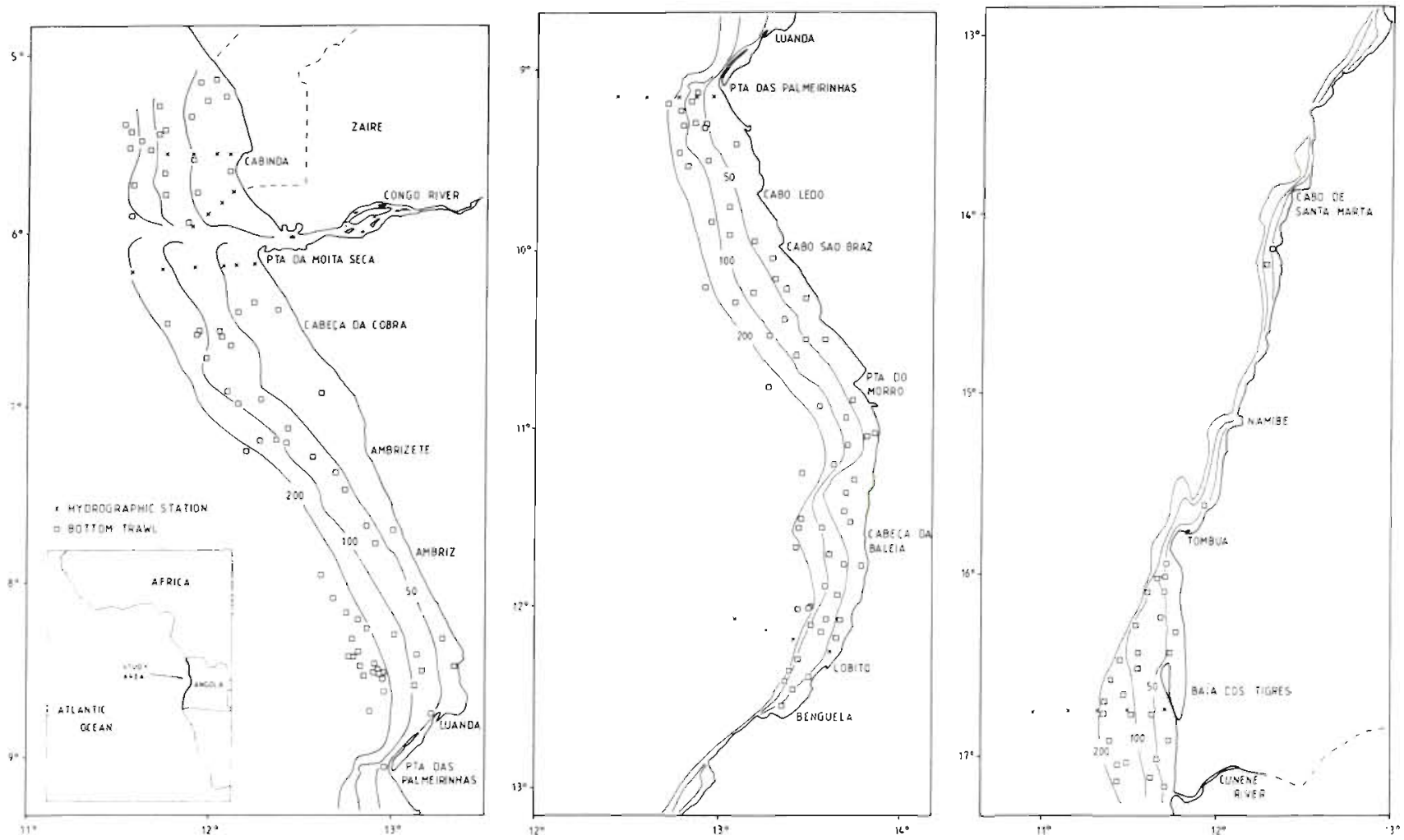

Fig. 1. Position of trawl hauls and hydrographic stations. February and March 1989 


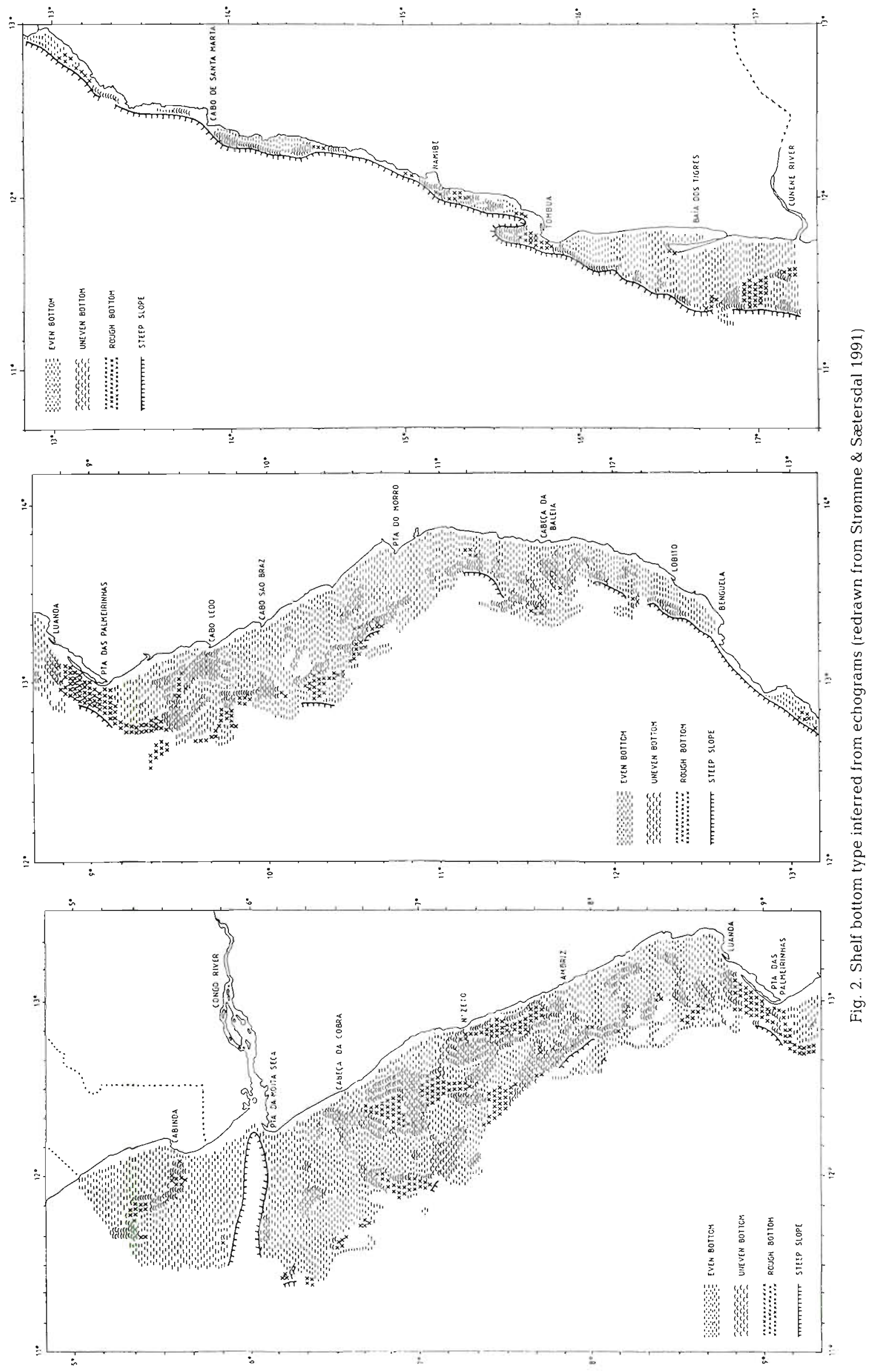



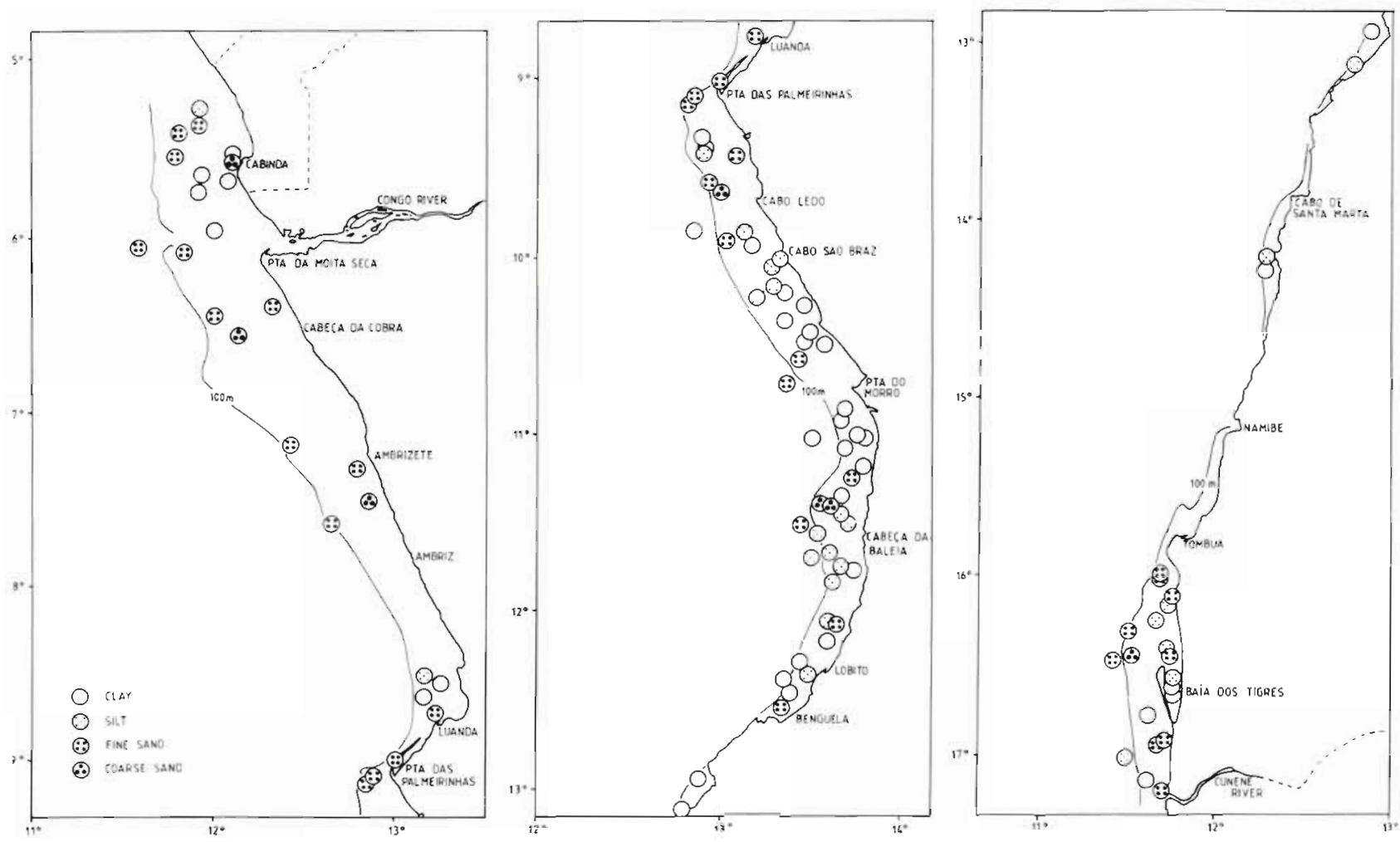

Fig. 3. Position of bottom samples and type of sediment. February and March 1989

During the austral summer (January to April; Fig. 4) the northern region (Cabinda to Pta. das Palmeirinhas) is characterized by a very shallow and marked thermocline, its upper boundary being found at about $10 \mathrm{~m}$ depth in the north and becoming deeper (between 25 and $50 \mathrm{~m}$ depth) southward. In the northern part of the area the halocline is also very sharp, mainly because of the increased rainfall and the increased runoff from the Congo River. The upper water layer consists of Equatorial Water, observed to 13 to $14^{\circ} \mathrm{S}$, characterized by low salinity, high temperature and reaching 30 to $40 \mathrm{~m}$ in thickness (Wauthy 1977). Oxygen levels usually above $2 \mathrm{ml} \mathrm{l}^{-1}$ are found to about $100 \mathrm{~m}$ depth, decreasing to slightly over $1 \mathrm{ml} \mathrm{l}^{-1}$ to the shelf edge. Between Pta. das Palmeirinhas and Benguela there is also a sharp thermocline between about 25 and $50 \mathrm{~m}$ depth and surface temperatures gradually decrease toward the south. Surface temperatures of the northern part (to Benguela), are usually 27 to $28^{\circ} \mathrm{C}$. Bottom temperatures of $20^{\circ} \mathrm{C}$ or more are found to about $50 \mathrm{~m}$ depth from Cabinda to Lobito. The southernmost part of the shelf, between Tombua and Cunene and particularly between 14 and $16^{\circ} \mathrm{S}$, is characterized by the presence of the permanent frontal system (convergence zone) between the southward flowing Angola Current and the north-moving surface waters of the Benguela Current. The front shifts seasonally through ca $2^{\circ}$ latitude.
The mechanisms responsible for maintaining the front within a relatively narrow range of latitudes seem to be, among others, the coastline orientation, bathymetry and wind stress (Shannon et al. 1987). During the austral summer the front is usually located further south, between about 16 and $18^{\circ} \mathrm{S}$. The front represents the southern limit of the waters of tropical/equatorial origin, with a sharp, almost permanent thermocline and the cold waters of the Benguela Current, with coastal, permanent upwelling, which is however moderate or weak in this season. South of Tombua temperatures near the bottom are always lower than $20^{\circ} \mathrm{C}$.

During the winter, with the strengthening of the southeast trade winds, a northward flowing coastal current develops, with upwelling occurring all along the coast. This phenomenon appears to be well developed especially off Pta. das Palmeirinhas and Lobito, and in correspondence with the capes (i.e. Cabo Ledo, Cabeça da Baleia and Pta do Morro). Surface temperatures of the northern region (from Cabinda to Lobito) are much lower, 20 to $22^{\circ} \mathrm{C}$, than in the summer. The thermocline is lifted and often broken down by the occurrence of upwelling. In the southern part (Tombua to Cunene) upwelling is at its peak, with surface temperatures near the coast down to $15^{\circ} \mathrm{C}$. Oxygen values $<2 \mathrm{ml} \mathrm{l}^{-1}$ are found from about $50 \mathrm{~m}$ depth and values below $1 \mathrm{ml} \mathrm{l}^{-1}$ are found at $100 \mathrm{~m}$ depth. 

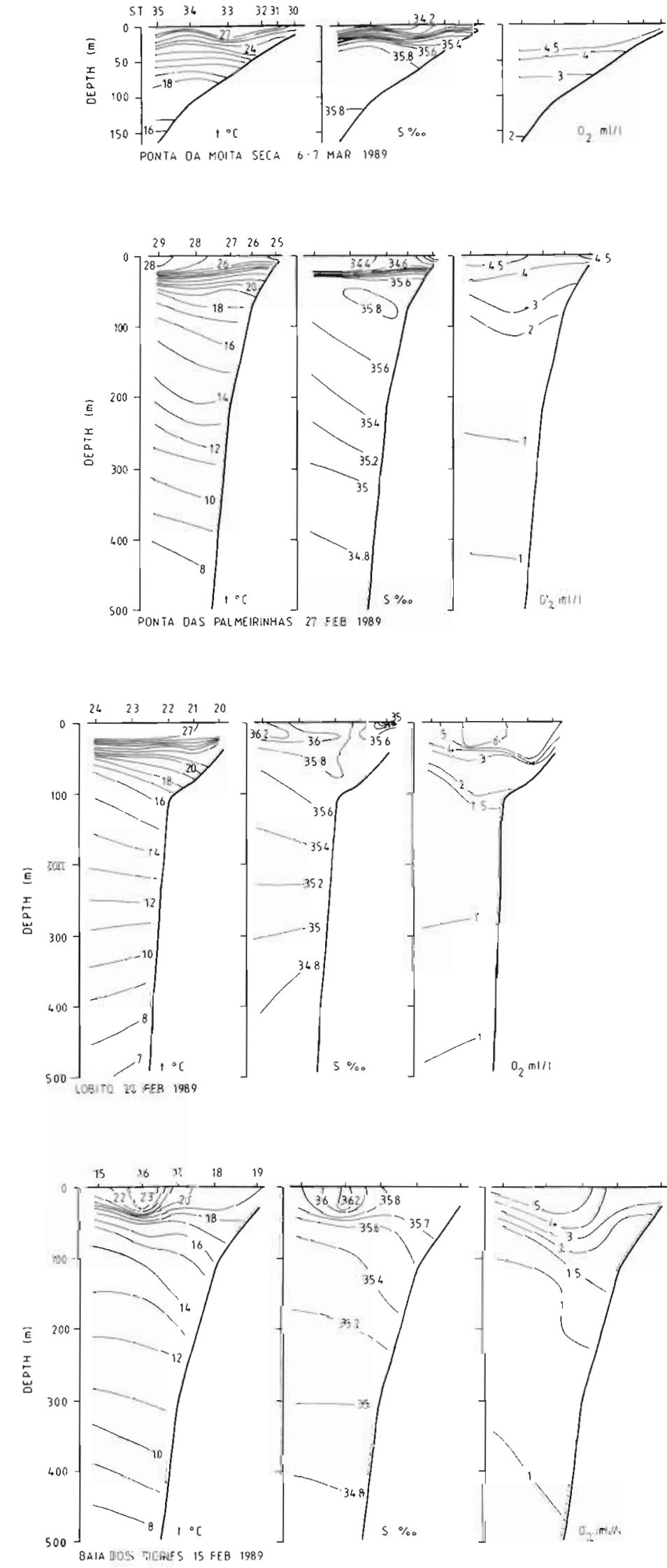

Berrit (1976) suggests that the upwelling off Gabon and Angola is not of Ekman-type because its occurrence corresponds with the time of minimum strength of the winds favourable to upwelling. Also, good correlation was found with wind strength in the western Atlantic. Voituriez \& Herbland (1982) discuss the different mechanisms that might be responsible for the eastern tropical Atlantic upwelling, including the increase in wind stress in the western Atlantic generating a Kelvin wave along the Equator in the west-east direction. They however conclude that it is not possible, based on present knowledge, to draw conclusions on which mechanism is really responsible for these upwellings.

Two different highly productive systems can be identified in Angolan waters: seasonal coastal upwelling, typifying mostly the northern and central parts southward to Tombua, and the almost permanent upwelling in the southern part of the area coinciding with the northernmost extension of the Benguela Current.

Other factors contributing to the enrichment in nutrients of the marine waters of Angola include the discharge from the Congo River and shelf-break upwelling. This phenomenon is common both in the tropics and elsewhere and reported as striking in the Gulf of Guinea (Longhurst \& Pauly 1987) and possibly responsible for enhanced production at the shelf-break area.

\section{MATERIAL AND METHODS}

Trawl data. Material was collected in the summer season (13 February to 16 March). A shrimp and fish trawl was used, with headline of $31 \mathrm{~m}$, footrope of $47 \mathrm{~m}$ and estimated headline height and distance between wings during towing of 6 and 18 to $20 \mathrm{~m}$ respectively. Mesh size was $2 \mathrm{~cm}$, with double lining in the cod end.
Fig. 4. Hydrographic profiles of temperature, salinity and oxygen at selected places along the Angolan coast (see also Fig. 1). February and March 1989 (redrawn from Strømme \& Sæetersdal 1991) 
Each tow had a standard duration of $30 \mathrm{~min}$ (other details in Strømme \& Sætersdal 1991). The bottom trawl stations were randomly set along the cruise track. A total of 167 stations were sampled in the course of the above survey (Fig. 1). Of these, 4 stations considered as 'non-valid' (because of gear damage) were not included in the analysis.

Each specimen caught was identified, counted and weighed separately. The FAO species identification sheets for fishery purposes, Fishing Areas 34/47 (in part) (Fisher et al. 1981) and the Guide to the commercial marine and brackish waters of Angola (Bianchi 1986) were used for identifying the species. Congeneric species which were difficult to separate were pooled together. All station and species data were stored using the B-trieve file system (data available in ASCII format upon request to the author and with the authorization of Angolan authorities).

Hydrographic data. Samples for temperature, salinity and oxygen were taken using Nansen bottles at standard depths and along fixed transects (Fig. 1). In the present analysis, the values of these variables at each station were inferred from the nearest hydrographic station.

Data analysis. Analysis was performed with the help of multivariate analytical techniques, i.e. a classification method, Two-Way Indicator species Analysis (TWIA; Hill 1979), implemented by the program TWINSPAN, and an ordination method, Detrended Correspondence Analysis (DCA; Hill \& Gauch 1980), implemented by the program DECORANA. The former is a divisive method that classifies sites and species and produces a sorted species by station table. Detrended Correspondence Analysis produces an ordination of the stations based on the abundance values of the species. The ordination summarizes multivariate data in a scatter, low-dimensional diagram and it is also useful for detecting possible outliers. Furthermore, the DCA version used for this study also correlates the main gradients (axes) with given environmental variables (ter Braak 1987). As a result of the analysis, means and standard deviations of the environmental variables are also produced for each group identified. A discussion on the validity of the above methods for this type of study is presented in Bianchi (1991).

A table of 'pseudo- $F$ ' values (ratios of the amonggroup to within-group variances) was made to evaluate the degree of conformity of a species to a sitegroup obtained from the above methods. A formal $F$-test cannot be performed in this case because it would be based on the same data previously used to establish the groups (Green \& Vascotto 1978).

In this study biomass (wet wt) was used as a measure of abundance. Each weight $(x)$ was converted to $\ln (x+1)$ before analysis with DCA and for calculating the 'pseudo- $F$ ' values. This transformation minimizes the dominant effect of anomalous catches. The addition of 1 unit is necessary to avoid problems derived by the presence of values $=0$ or $<1$. No transformation is necessary in the case of TWIA, where abundances are converted to numbers corresponding to different abundance classes ('pseudospecies'). In this study 5 pseudospecies were used, corresponding to classes with lower limits set at 0,10,100,1000 and $10000 \mathrm{~kg}$.

Demersal biomass densities (weight per unit area) were calculated using the 'swept-area' method by depth stratum:

$$
D_{j}=C_{j} / q a_{j}
$$

where $D_{\mathrm{j}}=$ density in Stratum $j$ [tons (n mile $)^{-2}$ ]; $C_{\mathrm{j}}=$ catch taken in hauls in Stratum $j$ (tons); $a_{j}=$ surface of the bottom 'swept' by the trawl hauls in Stratum $j$ (n mile $\left.{ }^{2}\right) ; q=$ catchability coefficient $(=1$, i.e. all fish in the path of the trawl were caught)

In the swept-area analysis, shallow-water pelagic species caught in the bottom trawl were not excluded. It is indeed quite difficult to differentiate between pelagic and demersal for the shallow-water species. Small pelagic fish of this depth zone are often found quite close to the bottom; some of them feed on bottom detritus and are preyed upon by both demersal and pelagic predators. Pelagic species of the deeper shelf were instead excluded from this analysis.

\section{RESULTS}

A total of 289 species comprising 3377403 specimens $(79964 \mathrm{~kg})$ were sampled in February and March 1989. Table 1 gives the list of the most important species collected and used in the analysis.

Appendix 1 shows the results from TWIA and Fig. 5 the dendrogram representing the relationships between the various groups (assemblages). The first division separates the deep water groups ( 7 and 8 ) from the shelf groups ( 1 to 6 ). At the second division level the shallow water assemblages (Groups 1 to 3) separate from the assemblages of the deeper shelf (Groups 4 to 6 ) while the 2 upper slope assemblages separate from each other also according to depth strata. At the third division level the assemblage of shallow waters (1) separates from Groups 2 and 3, found in slightly deeper waters, while the deeper shelf Groups 4 and 5 (from Cabinda to Benguela) separate from the corresponding assemblage of the Tombua-Cunene region (Group 6).

Fig. 6 shows the plot of stations on the first 2 DCA axes. The eigenvalues of the first 4 axes are $0.86,0.46$, 0.38 and 0.30 respectively, which shows that the 
Table 1. Main species collected in 1989 off Angola, by major taxonomic groups and families

\begin{tabular}{|c|c|}
\hline Cephalopods & Ogcocephalidae \\
\hline Loliginidae & Dibranchus atlanticus Peters, 1875 \\
\hline Alloteuthis africana Adam, 1950 & Ophidiidae \\
\hline Loligo vulgaris Lamark, 1798 & Brotula barbata (Bloch) in Bloch \& Schneider, 1801 \\
\hline Lolliguncola mercatoris Adam, 1941 & Monomitopus spp. \\
\hline Ommastrephidae & Merlucciidae \\
\hline Illex coindetii (Verany, 1837) & Merluccius capensis Castelnau, 1861 \\
\hline Todaropsis eblanae (Ball, 1841) & Merluccius paradoxus Franca, 1960 \\
\hline Sepiidae & Merluccius polli Cadenat, 1950 \\
\hline Sepia officinalis Linnaeus, 1758 & Moridae \\
\hline Decapod crustaceans & $\begin{array}{l}\text { Laemonema spp. } \\
\text { Physiculus spp. }\end{array}$ \\
\hline $\begin{array}{l}\text { Solenoceridae } \\
\text { Solenocera africana Stebbing, } 1917\end{array}$ & $\begin{array}{l}\text { Macrouridae } \\
\text { Coelorinchus coelorhincus (Risso, 1810) }\end{array}$ \\
\hline Aristeidae & Hymenocephalus italicus Giglioli, 1884 \\
\hline Aristeus varidens Holthuis, 1952 & Malacocephalus laevis (Lowe, 1843) \\
\hline Plesiopenaeus edwardsianus (Johnson, 1867) & Malacocephalus occidentalis Goode \& Bean, 1885 \\
\hline Penaeidae & Nezumia aequalis (Günther, 1878) \\
\hline Parapenaeopsis atlantica Balss, 1914 & Zeidae \\
\hline Parapenaeus longirostris (Lucas, 1846) & Zenopsis conchifer (Lowe, 1852) \\
\hline Penaeus notialis Pérez-Farfante, 1967 & Zeus faber Linnaeus, 1758 \\
\hline Nematocarcinidae & Fistulariidae \\
\hline Nematocarcinus africanus Crosnier \& Forest, 1973 & Fistularia petimba (Lacepède, 1803) \\
\hline Palaemonidae & Scorpaenidae \\
\hline Nematopalaemon hastatus (Aurivillius, 1898) & Pontinus spp. \\
\hline Geryonidae & Triglidae \\
\hline Geryon maritae Manning and Holthuis, 1981 & $\begin{array}{l}\text { Chelidonichthys capensis (Cuvier in Cuv. \& Val.,1829) } \\
\text { Chelidonichthys gabonensis (Poll \& Roux. 1955) }\end{array}$ \\
\hline Sharks & Chelidonichthys lastoviza (Bonnaterre. 1788) \\
\hline Squalidae & Lepidotrigla Cadmani Regan, 1915 \\
\hline Centrophorus granulosus (Bloch \& Schneider, 1801) & Lepidotrigla carolae Richards, 1968 \\
\hline Etmopterus spp. & Trigla lyra Linnaeus, 1758 \\
\hline Squatinidae & Peristediidae \\
\hline Squatina oculata Bonaparte, 1840 & Peristedion cataphractum Linnaeus, 1758 \\
\hline Triakidae & Serranidae \\
\hline Mustelus mustelus (Linnaeus, 1758) & $\begin{array}{l}\text { Epinephelus aeneus (Geoffroy Saint-Hilaire, 1809) } \\
\text { Epinephelus alexandrinus (Valenciennes, 1828) }\end{array}$ \\
\hline Batoid fishes & Antiidae \\
\hline Rajidae & Anthias anthias (Linnaeus, 1758) \\
\hline Raja miraletus Linnaeus, 1758 & Acropomatidae \\
\hline Bony fishes & Synagrops microlepis Norman, 1935 \\
\hline Albulidae & Branchiostegidae \\
\hline Albula vulpes (Linnaeus, 1758) & Branchiostegus semifasciatus (Norman, 1931) \\
\hline Pterothrissus belloci Cadenat, 1937 & Carangidae \\
\hline Clupeidae & Chloroscombrus chrysurus (Linnaeus, 1766) \\
\hline Ilisha africana (Bloch, 1795) & Decapterus punctatus (Cuvier, 1829) \\
\hline Sardinella aurita Valenciennes, 1847 & Decapterus rhonchus (Geoffroy Saint-Hilaire, 1817) \\
\hline Sardinella maderensis (Lowe, 1839) & Selar crumenophthalmus (Bloch, 1793) \\
\hline Sardinops ocellata (Pappé, 1853) & Selene dorsalis (Gill, 1862) \\
\hline Engraulididae & Trachurus capensis Castelnau, 1861 \\
\hline Engraulis encrasicolus (Linnaeus, 1758) & Trachurus trecae Cadenat, 1949 \\
\hline Ariidae & Centracanthidae \\
\hline Arius parkii Günther, 1864 & Spicara alta (Osorio, 1917) \\
\hline Myctophidae & Spicara nigricauda (Norman, 1931) \\
\hline & Haemulidae \\
\hline $\begin{array}{l}\text { Synodontidae } \\
\text { Saurida brasiliensis Norman } 1935\end{array}$ & Brachydeuterus auritus (Valenciennes, 1831) \\
\hline Saunda brasiliensis Norman, 1935 & Pomadasys incisus (Bowdich, 1825) \\
\hline Chlorophthalmidae & Pomadasys jubelini (Cuvier, 1830) \\
\hline Chlorophthalmus atlanticus Poll, 1953 & Pomadasys peroteti (Cuvier, 1830 ) \\
\hline
\end{tabular}


Table 1 (continued)

Sparidae

Boops boops (Linnaeus, 1758)

Dentex angolensis Poll \& Maul, 1953

Dentex barnardi (Cadenat, 1970)

Dentex canariensis Steindachner, 1881

Dentex congoensis Poll, 1954

Dentex gibbosus (Rafinesque, 1810)

Dentex macrophthalmus (Bloch, 1791)

Lithognathus mormyrus (Linnaeus, 1758)

Pagellus bellottii Steindachner, 1882

Sparus auriga (Valenciennes, 1843)

Sparus caeruleostictus (Valenciennes, 1830)

Sparus pagrus africanus Akazaki, 1962

Sciaenidae

Argyrosomus hololepidotus (Lacepède, 1802)

Atractoscion aequidens (Cuvier, 1830)

Pentheroscion mbizi (Poll, 1950)

Pseudotolithus senegalensis (Valenciennes, 1833)

Pseudotolithus typus Bleeker, 1863

Pteroscion peli (Bleeker, 1863)

Umbrina canariensis Valenciennes, 1843

Mullidae

Pseudupeneus prayensis (Cuvier, 1829)

Sphyraenidae

Sphyraena guachancho Cuvier, 1829

Sphyraena sphyraena (Linnaeus, 1758)
Polynemidae

Galeoides decadactylus (Bloch, 1795)

Uranoscopidae

Uranoscopus albesca Regan, 1915

Scombridae

Scomberomorus tritor (Cuvier, 1831)

Trichiuridae

Benthodesmus tenuis (Günther, 1877)

Lepidopus caudatus (Euphrasen, 1788)

Trichiurus lepturus Linnaeus, 1758

Stromateidae

Stromateus fiatola Linnaeus, 1758

Ariommidae

Ariomma bondi Fowler, 1930

Citharidae

Citharus linguatula (Linnaeus, 1758)

Bothidae

Arnogiossus imperialis (Rafinesque, 1810)

Soleidae

Dicologoglossa cuneata (de la Pylaie Moreau, 1881)

Tetraodontidae

Lagocephalus laevigatus (Linnaeus, 1766)

Balistidae

Balistes capriscus Gmelin, 1788 gradient represented by the first axis is by far the most important. Table 2 shows the correlation of DCA Axes 1 to 4 with the environmental variables and with latitude. Depth, temperature and oxygen are strongly correlated with DCA Axis $1(\mathrm{r}=0.90,-0.97$ and -0.89 respectively). Axis 2 is significantly correlated only with latitude $(r=-0.75, p<0.05)$.

Fig. 7 shows the results from a further analysis with DCA on the deeper shelf assemblages (Groups 4 to 6). The eigenvalues are $0.53,0.34,0.23$ and 0.18 for the first DCA axes, respectively. Table 3 shows the corre-

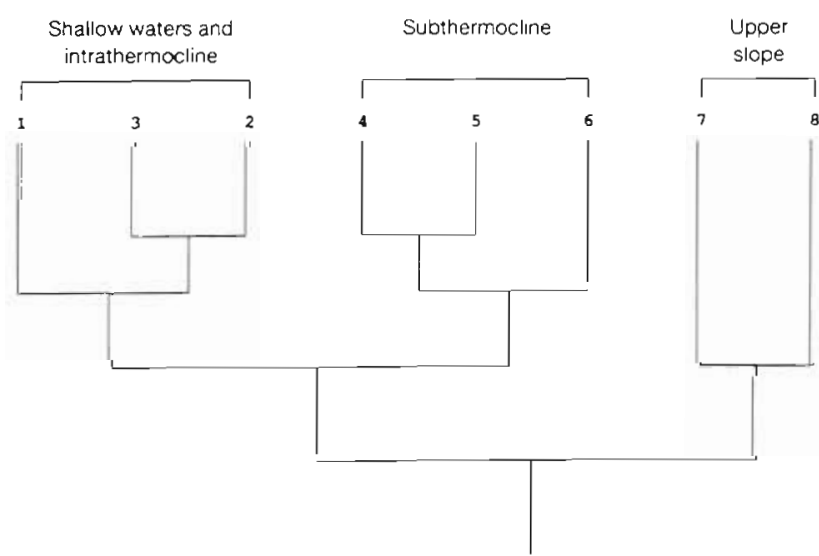

Fig. 5. Dendrogram of Station Groups 1 to 8 derived from classification with the program TWINSPAN (Hill 1979). See 'Results' for description of each station group lation of Axes 1 and 2 with the environmental variables and with latitude. Axis 1 is highly correlated with latitude $(r=-0.86)$, but significant correlation $(p<0.05)$ is also found with temperature, salinity and oxygen. Axis 2 shows significant correlation with depth.

Table 4 presents results from the 'pseudo- $F$ ' analysis and Table 5 the weight, numbers and frequency of the main species in each group.

Fig. 8 shows the position of the stations after having been assigned to each group. The plot of mean biomass densities by depth stratum for northern, central and southern Angola is presented in Fig. 9 while Table 6 gives the number of stations sampled by depth stratum.

Below are descriptions of the 8 groups identified.

\section{Group 1 - Shallow water assemblage, from northern Angola to Benguela}

The 15 stations included in this group have an average depth of $24 \mathrm{~m}$, temperature $23^{\circ} \mathrm{C}$ and oxygen levels usually high, $3.7 \mathrm{ml} \mathrm{l^{-1 }}$ on average. The species caught here are those typically found in the warm and turbid waters above the thermocline, often associated with river mouths, able to tolerate low salinities and on soft, mud bottoms. The 'pseudo- $F$ ' table (Table 4) shows the species characteristic of this group. Among these are the drum Pteroscion peli, the croaker Pseudotolithus senegalensis, the butterfish Stromateus fiatola, the African threadfin Galeoides decadactylus and the 
Fig. 6. Detrended correspondence analysis of bottom-trawl stations in the February-March survey 1989 (SD units $\times 10$ ). Corresponding TWIA (Two-Way Indicator species analysis) Groups 1 to 8 can be recognized by the different symbols

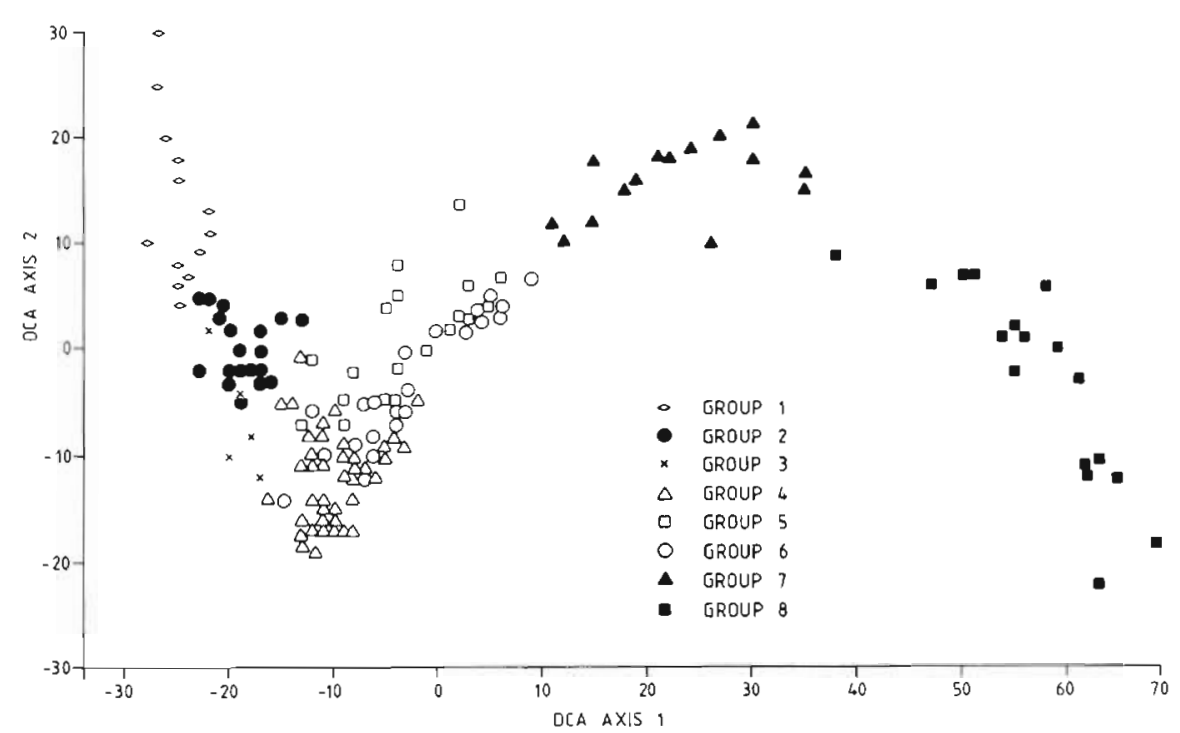

Table 2. Pearson product-moment correlation coefficient between sample scores on DCA (Detrended Correspondence Analysis) Axes 1 and 2 and environmental variables for all stations. Values with asterisk indicate significant correlation $(\mathrm{p}<0.05, \mathrm{df}=161)$

\begin{tabular}{lrr|}
\hline Variable & Axis 1 & Axis 2 \\
\hline Depth & $0.90^{\circ}$ & -0.04 \\
Temperature & $-0.97^{\circ}$ & 0.09 \\
Salinity & $-0.48^{\circ}$ & -0.08 \\
Oxygen & $-0.89^{\circ}$ & 0.18 \\
Latitude & -0.06 & $-0.75^{\circ}$ \\
\hline
\end{tabular}

Table 3. Pearson product-moment correlation coefficient between sample scores on DCA (Detrended Correspondence Analysis) Axes 1 and 2 and environmental variables for the subthermocline shelf stations. Values with asterisk indicate significant correlation ( $\mathrm{p}<0.05, \mathrm{df}=82$ )

\begin{tabular}{|lcc|}
\hline Variable & Axis 1 & Axis 2 \\
\hline Depth & 0.14 & $0.55^{\circ}$ \\
Temperature & $-0.49^{\circ}$ & -0.32 \\
Salinity & $-0.50^{\circ}$ & -0.20 \\
Oxygen & $-0.65^{\circ}$ & -0.12 \\
Latitude & $-0.86^{\circ}$ & -0.34 \\
\hline
\end{tabular}

Fig. 7. Detrended correspondence analysis of intermediate-shelf bottom-trawl stations February-March survey 1989 (SD units $\times$ 10). Corresponding TWIA (Two-Way Indicator species Analysis) Groups 4 to 6 can be recognized by the different symbols

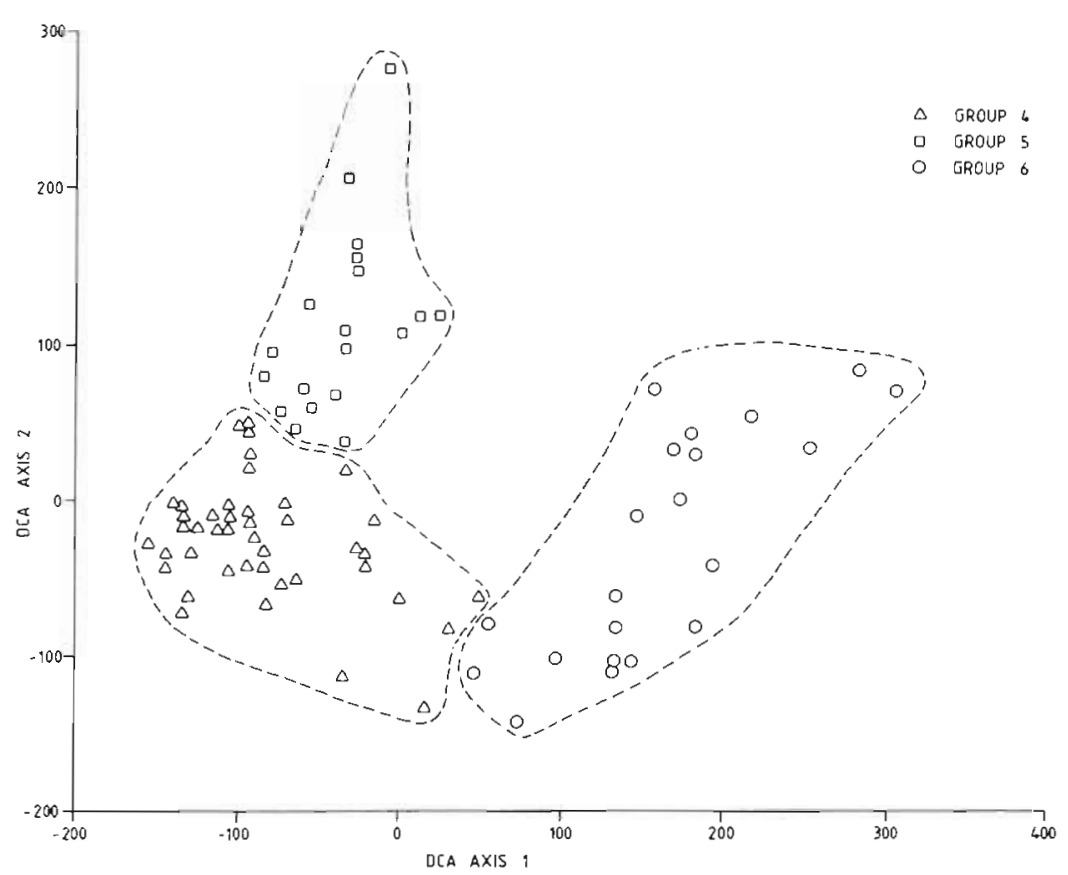


Table 4. Two-way table based on classification and ordination analyses, showing conforming species groups within site groups Pseudo-F $(\mathrm{P}-F)$ values preceded by an asterisk indicate conformity at a significance of $p=0.05$ or better. The average biomass value $(\mathrm{kg})$ of a species within each group, converted to $\ln (x+1)$, is preceded by an asterisk whenever the $95 \%$ confidence interval for the mean is not overlapping. ( ..) indicates that a species is found only in 1 group. Mean values of environmental variables

are also shown for each group, with standard deviations (in parentheses). Only the most important species are included

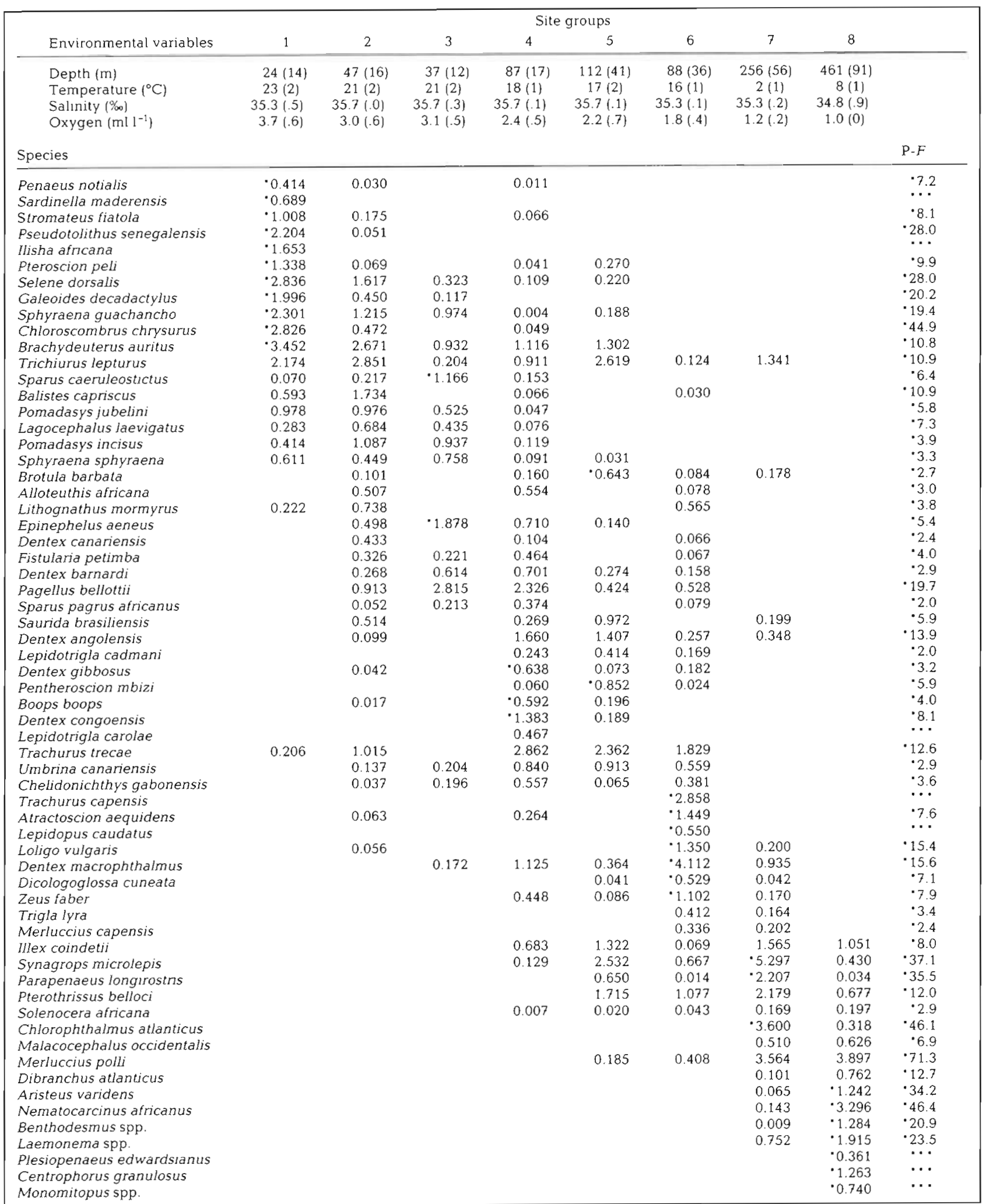


Table 5. Total weight $(\mathrm{W} ; \mathrm{kg})$, numbers $(\mathrm{N})$ and frequency $(\mathrm{F} ; \mathrm{no}$. of stations where found in respective group) of main species from Station Groups 1 to 8

\begin{tabular}{|c|c|c|c|c|c|c|c|c|c|c|c|}
\hline Species & w & $(\%)$ & $\mathrm{N}$ & $(\%)$ & $\mathrm{F}$ & Species & W & $(\%)$ & $\mathrm{N}$ & $(\%)$ & $\mathrm{F}$ \\
\hline Group 1 (15 stations) & & & & & & Sepia officinalis & 80 & (0) & 258 & (0) & 8 \\
\hline Brachydeuterus auritus & 2214 & $(21)$ & 86498 & (14) & 13 & Fistularia petimba & 54 & (0) & 152 & (0) & 19 \\
\hline Selene dorsalls & 1602 & (16) & 12700 & (2) & 15 & Zeus faber & 50 & (0) & 220 & (0) & 17 \\
\hline Tnchiurus lepturus & 1193 & (12) & 9138 & (2) & 10 & Lepidotrigla cadmanı & 31 & (0) & 477 & (0) & 11 \\
\hline Sphyraena guachancho & 719 & (7) & 1364 & (0) & 11 & Sparus pagrus africanus & 25 & (0) & 43 & (0) & 4 \\
\hline Pseudotolithus senegalensis & 682 & (7) & 2462 & (0) & 9 & Total & 14036 & (89) & 448331 & $\{95\}$ & \\
\hline Chloroscombrus chrysurus & 647 & (6) & 11254 & (2) & 13 & Total (all species) & 15817 & & 470430 & & \\
\hline Ilisha africana & 587 & (6) & 31704 & (5) & 9 & & & & & & \\
\hline Galeoides decadactylus & 567 & (5) & 13278 & (2) & 10 & Group 5 (19 stations) & & & & & \\
\hline Sphyraena sphyraena & 416 & (4) & 14596 & (2) & 4 & Synagrops microlepis & 4502 & (52) & 385464 & (82) & 11 \\
\hline Engraulis encrasicolus & 270 & (3) & 125563 & (21) & 3 & Trichiurus lepturus & 826 & (9) & 7436 & (2) & 17 \\
\hline Pteroscion peli & 149 & (1) & 3622 & $(1)$ & 7 & Trachurus trecae & 813 & (9) & 22313 & (5) & 16 \\
\hline Balistes capriscus & 108 & (1) & 503 & (0) & 3 & Brachydeuterus auritus & 532 & (6) & 7500 & (2) & 7 \\
\hline Pomadasys jubelini & 100 & (1) & 248 & (0) & 5 & Pterothrissus belloci & 323 & (4) & 3148 & (1) & 15 \\
\hline Nematopalaemon hastatus & 82 & (1) & 277440 & (46) & 1 & Pentheroscion mbizi & 163 & (2) & 2334 & (0) & 7 \\
\hline Stromateus fiatola & 75 & (1) & 201 & (0) & 7 & Illex coindetii & 148 & (2) & 3169 & (1) & 13 \\
\hline Sardinella maderensis & 22 & (0) & 436 & (0) & 11 & Dentex angolensis & 107 & (1) & 663 & (0) & 14 \\
\hline Penaeus notialis & 15 & (0) & 322 & (0) & 5 & Umbrina canariensis & 102 & (1) & 397 & (0) & 8 \\
\hline Total & 9448 & (92) & 591329 & (97) & & Brotula barbata & 95 & (1) & 123 & (0) & 8 \\
\hline \multirow[t]{2}{*}{ Total (all species) } & 10317 & & 601273 & & & Saurida brasiliensis & 73 & (1) & 9653 & (2) & 13 \\
\hline & & & & & & Parapenaeus longurostris & 50 & (1) & 12716 & (3) & 14 \\
\hline Group 2 (21 stations) & & & & & & Dentex macrophthalmus & 33 & (0) & 288 & (0) & 3 \\
\hline Brachydeuterus auritus & 3779 & (43) & 120227 & (60) & 15 & Dentex barnardi & 27 & (0) & 46 & (0) & 4 \\
\hline Trichiurus lepturus & 1922 & (23) & 36930 & (18) & 17 & Lepidotrigla cadmani & 21 & (0) & 184 & (0) & 6 \\
\hline Pomadasys jubelini & 805 & (9) & 1568 & (1) & 7 & Pagellus bellotti & 18 & (0) & 100 & (0) & 7 \\
\hline Balistes capriscus & 673 & (8) & 3161 & (2) & 11 & Total & 7833 & (90) & 455534 & (97) & \\
\hline Pomadasys incisus & 376 & (4) & 2084 & (1) & 7 & Total (all species) & 8671 & & 470875 & & \\
\hline Selene dorsalis & 206 & (2) & 684 & (0) & 15 & & & & & & \\
\hline Sphyraena guachancho & 125 & (1) & 249 & (0) & 12 & Group 6 (24 stations) & & & & & \\
\hline Trachurus trecae & 123 & (1) & 16166 & (8) & 12 & Dentex macrophthalmus & 6853 & (39) & 286791 & (46) & 20 \\
\hline Pagellus bellottii & 106 & (1) & 862 & (0) & 12 & Trachurus capensls & 5612 & (32) & 132867 & (21) & 21 \\
\hline Lithognathus mormyrus & 102 & (1) & 424 & (0) & 7 & Trachurus trecae & 1156 & (7) & 116275 & (19) & 18 \\
\hline Epinephelus aeneus & 69 & (1) & 14 & (0) & 4 & Atractoscion aequidens & 468 & (3) & 337 & (0) & 12 \\
\hline Alloteuthis africana & 41 & (0) & 9412 & (5) & 10 & Lepidopus caudatus & 438 & (2) & 4902 & (1) & 3 \\
\hline Lagocephalus laevigatus & 37 & (0) & 62 & (0) & 11 & Spicara alta & 424 & (2) & 13648 & (2) & 3 \\
\hline Dentex canarjensis & 33 & (0) & 100 & (0) & 5 & Pterothrissus belloci & 420 & (2) & 4809 & (1) & 9 \\
\hline Galeoides decadactylus & 31 & (0) & 65 & (0) & 7 & Synagrops microlepis & 271 & (1) & 23737 & (4) & 5 \\
\hline Sphyraena sphyraena & 31 & (0) & 94 & (0) & 7 & Loligo vulgaris & 201 & (1) & 18444 & (3) & 15 \\
\hline Chloroscombrus chrysurus & 21 & (0) & 1528 & (1) & 11 & Umbrina canariensis & 156 & (1) & 974 & (아 & 6 \\
\hline Fistularia petimba & 21 & (0) & 67 & (0) & 4 & Lithognathus mormyrus & 147 & (1) & 502 & (0) & 6 \\
\hline Dentex barnardi & 12 & (0) & 85 & $(0)$ & 5 & Zeus faber & 142 & (1) & 302 & (0) & 13 \\
\hline Total & 8513 & (96) & 193782 & (96) & & Pagellus bellottii & 68 & (0) & 540 & (0) & 8 \\
\hline \multirow{2}{*}{ Total (all species) } & 8814 & & 200087 & & & Chelidonichthys gabonensis & 65 & (0) & 616 & 101 & 4 \\
\hline & & & & & & Merluccius polli & 44 & (0) & 1084 & (0) & 6 \\
\hline Group 3 (5 stations) & & & & & & Trigla lyra & 39 & (0) & 115 & (0) & 5 \\
\hline Pagellus bellottii & 207 & (38) & 1185 & (18) & 5 & Dicologoglossa cuneata & 35 & (0) & 522 & (0) & 10 \\
\hline Pomadasys incisus & 105 & (19) & 450 & (7) & 1 & Total & 16539 & (94) & 606465 & (97) & \\
\hline Epinephelus aeneus & 47 & (9) & 20 & (0) & 4 & Total (all species) & 17593 & & 621992 & & \\
\hline Brachydeuterus auritus & 26 & (5) & 4606 & (68) & 2 & & & & & & \\
\hline Dentex barnardi & 21 & (4) & 46 & (1) & 1 & Group 7 (15 stations) & & & & & \\
\hline Sphyraena guachancho & 19 & (4) & 45 & (1) & 3 & Synagrops microlepis & 6175 & (52) & 364356 & (66) & 15 \\
\hline Sparus caeruleostictus & 16 & (3) & 27 & (0) & 4 & Chlorophthalmus atlanticus & 2646 & (22) & 72486 & (13) & 12 \\
\hline Fistularia petimba & 11 & (2) & 14 & (0) & 2 & Merluccius polli & 1212 & (10) & 14584 & (3) & 14 \\
\hline Sphyraena sphyraena & 9 & (2) & 41 & (1) & 3 & Dentex macrophthalmus & 367 & (3) & 2350 & (0) & 5 \\
\hline Pomadasys jubelini & 6 & (1) & 6 & (0) & 2 & Illex coindetil & 256 & (2) & 3835 & (1) & 10 \\
\hline Lagocephalus laevigatus & 4 & (1) & 14 & (0) & 2 & Parapenaeus longirostris & 254 & (2) & 53205 & (10) & 14 \\
\hline Selene dorsalis & 3 & (0) & 14 & (0) & 2 & Pterothrissus belloci & 233 & (2) & 1637 & (0) & 12 \\
\hline Trichiurus lepturus & 2 & (0) & 22 & (0) & 1 & Trichiurus lepturus & 189 & (2) & 904 & (0) & 8 \\
\hline Chelidonichthys gabonensis & 1 & (0) & 6 & (0) & 2 & Laemonema spp. & 84 & (1) & 1303 & (0) & 4 \\
\hline Total & 477 & (88) & 6496 & (96) & & Malacocephalus occidentahs & 37 & (0) & 341 & (0) & 3 \\
\hline \multirow[t]{2}{*}{ Total (all species) } & 540 & & 6750 & & & Total & 11453 & (96) & 515001 & (93) & \\
\hline & & & & & & Total (all species) & 11904 & & 552605 & & \\
\hline Group 4 ( 45 stations) & & & & & & & & & & & \\
\hline Trachurus trecae & 4514 & (28) & 180417 & $(38)$ & 37 & Group 8 (17 stations) & & & & & \\
\hline Dentex macrophthalmus & 2762 & (17) & 14529 & (3) & 12 & Merluccius polli & 2459 & (39) & 10224 & (2) & 16 \\
\hline Pagellus bellottii & 1395 & (9) & 16979 & (4) & 40 & Nematocarcinus africanus & 2009 & (32) & 391466 & (86) & 14 \\
\hline Dentex congoensis & 1068 & (7) & 29943 & (6) & 21 & Laemonema spp. & 207 & (3) & 3369 & (1) & 12 \\
\hline Brachydeuterus auritus & 835 & (5) & 36432 & (8) & 16 & Centrophorus granulosus & 150 & (2) & 38 & (0) & 7 \\
\hline Umbrina cariensis & 751 & (5) & 2506 & (0) & 15 & llex coindetii & 116 & (2) & 740 & (0) & 8 \\
\hline Trichiurus lepturus & 529 & (3) & 2236 & (0) & 19 & Benthodesmus tenuis & 114 & (2) & 3836 & (1) & 11 \\
\hline Dentex angolensis & 442 & (3) & 5543 & (1) & 34 & Pterothrissus belloci & 107 & (2) & 710 & (0) & 4 \\
\hline Epinephelus aeneus & 277 & (2) & 66 & (0) & 12 & Aristeus varidens & 67 & (1) & 3708 & (1) & 13 \\
\hline Boops boops & 228 & (1) & 12594 & (3) & 21 & Dibranchus atlanticus & 44 & (1) & 3144 & (1) & 13 \\
\hline Dentex barnardi & 217 & (1) & 563 & $(0)$ & 16 & Malacocephalus occidentalis & 30 & (1) & 416 & (0) & 7 \\
\hline Alloteuthis afncana & 197 & (1) & 99422 & (21) & 12 & Plesiopenaeus edwardsianus & 20 & (0) & 167 & (0) & 10 \\
\hline Dentex gibbosus & 186 & (1) & 201 & (0) & 11 & Synagrops microlepis & 19 & $(0)$ & 538 & (0) & 7 \\
\hline Saurida brasillensis & 163 & (1) & 35566 & (8) & 21 & Chlorophthalmus atlanticus & 12 & $(0)$ & 284 & $(0)$ & 6 \\
\hline Lepidotrigla carolae & 104 & (1) & 4854 & (1) & 14 & Solenocera africana & 6 & (0) & 621 & $(0)$ & 4 \\
\hline Illex coindetii & 99 & (1) & 4788 & (1) & 23 & Total & 5360 & (85) & 419261 & (93) & \\
\hline Chelidonichthys gabonensis & 85 & (0) & 542 & (0) & 18 & Total (all species) & 6308 & & 453391 & & \\
\hline
\end{tabular}


pink shrimp Penaeus notialis. Pelagic species usually associated with the above demersal fauna are the flat sardinella Sardinella maderensis, the West African ilisha llisha africand and the Atlantic bumper Chloroscombrus chrysurus. The highest biomass consists, however, of typically eurybathic and eurythermic species like the hairtail Trichiurus lepturus and the big-eye grunt Brachydeuterus auritus and 2 species also found in slightly deeper and cooler waters, like the guachanche barracuda Sphyraena guachancho and the African lookdown Selene dorsalis. These 4 species are the most abundant and make up $55 \%$ of the total catches from these stations (Table 5). A very large catch $(40000 \mathrm{~kg})$ of big-eye grunt between Pta. do Morro and Cabeça da Baleia was not included in the analysis because it is considered to be exceptional. The presence of this large concentration is possibly to be related to spawning activity. The shrimp Nematopalaemon hastatus, accounting for $46 \%$ of the catches in this group in numerical abundance, is a typically estuarine species known to occur throughout the Angolan coast. However, it was caught only once during this survey most probably because of its very shallow depth-distribution range and estuarine habitat preferences.

This assemblage largely coincides with the 'peuplement littoral' described by Durand (1967) for Congo and by Domain (1980) for the continental shelf off Senegal and Mauritania, as well as the 'estuarine and offshore sciaenid subcommunities' of the Gulf of Guinea described by Longhurst (1965) and Fager \& Longhurst (1968), of typically tropical nature. Its distribution along the Angolan coast broadly coincides with the presence of the Equatorial Water. This assemblage seems to be stable since the species composition is essentially the same as that described by those authors, despite the fishing activities of the last $20 \mathrm{yr}$.

\section{Group 2 - Coastal species, mainly in the thermocline area, from Luanda to Benguela}

This group of 21 stations was at an average depth of $47 \mathrm{~m}$, with temperature and oxygen values below the values found in shallow waters (about $21^{\circ} \mathrm{C}$ and $3 \mathrm{ml} \mathrm{l}^{-1}$ respectively). Several bottom samples showed that clay and silt substrate dominate this area, sometimes mixed with fine sand (Figs. 2 \& 3). Coarse sand was found just north of Cabeça da Baleia, at 40 and $50 \mathrm{~m}$ depth. The eurybathic Trichiurus lepturus and Brachydeuterus auritus dominate most stations both in weight and numbers. Most probably, because of their ability to live at different levels of the water column, they can most easily occupy the thermocline area
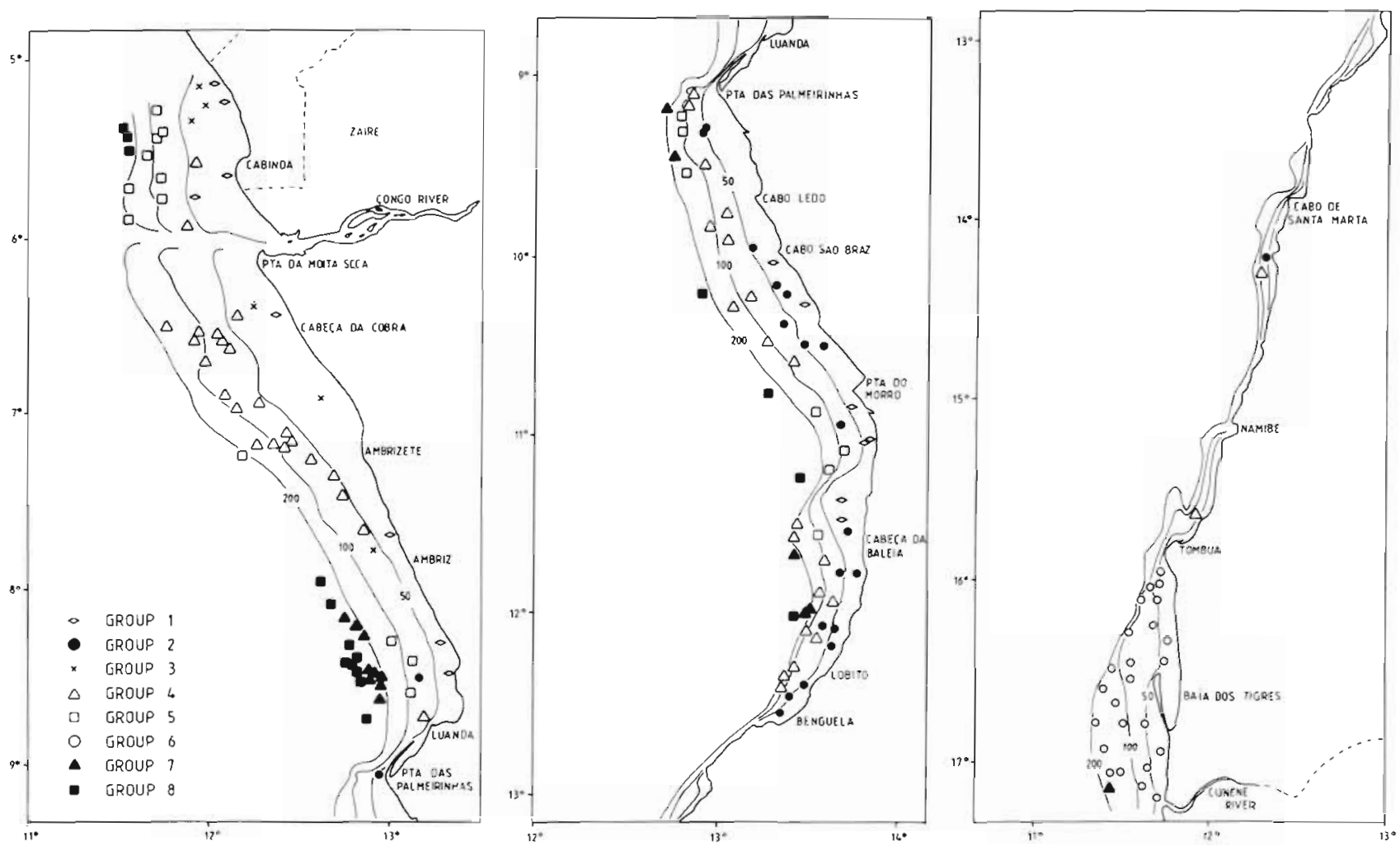

Fig. 8. Position of stations after being assigned to the different groups 

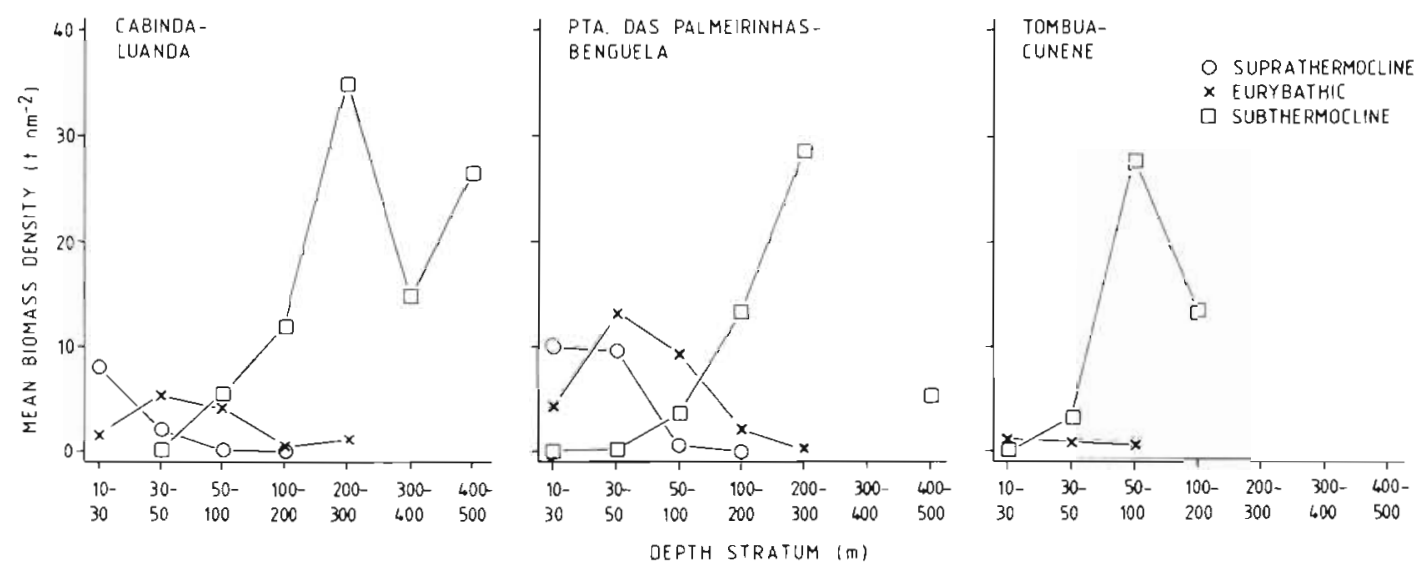

Fig. 9. Plot of mean biomass density by depth stratum from bottom trawl catches for northern, central and southern Angola (February and March 1989). The pelagic species Ilisha africana, Chlorocombrus chrysurus and Selene dorsalis are also included in the analysis (depth strata 10-30 and 30-50 m). For definition of species categories see 'Discussion - Biomass'

Table 6. Number of stations sampled by depth stratum for northern Angola (Cabinda-Luanda), central Angola (Pta. das Palmeirinhas-Benguela) and southern Angola (Tombua-Cunene)

\begin{tabular}{|lccccccc|}
\hline Location & \multicolumn{7}{c|}{ Depth stratum (m) } \\
& $10-30$ & $30-50$ & $50-100$ & $100-200$ & $200-300$ & $300-400$ & $400-500$ \\
\hline Cabinda-Luanda & 8 & 7 & 25 & 8 & 9 & 4 \\
Pta.Palmeirinhas-Benguela & 9 & 10 & 21 & 12 & 4 & 0 \\
Tombua-Cunene & 2 & 6 & 9 & 9 & 0 & 0 \\
\hline
\end{tabular}

characterized by a rapid change of the physical water conditions. The triggerfish Balistes capriscus is also a eurybathic species and an important element of this group. Selene dorsalis and Sphyraena guachancho, with a shallower depth distribution, and the red pandora Pagellus bellottii and the Cunene horse mackerel Trachurus trecae, with a deeper distribution range, were consistently caught at these stations. The grunt Pomadasys incisus and the striped seabream Lithognathus mormyrus gave relatively high catches in the Lobito-Benguela area.

\section{Group 3-Coastal species, in the thermocline area, in the northern part of the area, on sandy/hard bottoms}

This is a group of 5 stations with an average depth of about $37 \mathrm{~m}$, temperature of $21^{\circ} \mathrm{C}$, oxygen concentration of $3.1 \mathrm{ml} \mathrm{l}^{-1}$ and salinity $35.7 \%$, found off and north of Cabinda and south of the Congo River mouth to about Ambriz. This group is distinct from the other shallow water stations because of the presence of the bluespotted seabream Sparus caeruleostictus and the white grouper Epinephelus aeneus while all the species of Group 1 are present in small numbers or absent. Brachydeuterus auritus and Trichiurus lepturus are also present in very small numbers. Pagellus bellottii was also consistently caught at these stations.
This group also seems to belong to the tropical regime and is also found in the Gulf of Guinea and described by Fager \& Longhurst (1968) as an assemblage found at the bottom of the thermocline, mainly on hard bottoms, where the species that usually dominate the thermocline area are replaced by some members of the deeper sparid assemblage, probably because of the nature of the bottom.

Four bottom samples taken in this area showed the presence of fine and coarse sand. Also, the echograms showed the presence of rough bottoms and rocky outcrops in this area. This type of assemblage is most probably an important element of the Angola fish fauna but is poorly represented in our data because of the difficulty in using bottom trawls on rocky grounds.

The 3 groups which follow include species of the subthermocline assemblages of the continental shelf (50 to $150-200 \mathrm{~m}$ ). Two major subdivisions can be identified: an assemblage consisting, among others, of several species of Sparidae, with preference for sandy, fine sand to muddy bottoms, and an assemblage found on the shelf between Tombua and Cunene, and largely coinciding with the AngolaBenguela frontal system. The first group includes a subgroup with species with a clear preference for soft 
bottoms. Only 1 species, the Cunene horse mackerel Trachurus trecae, is abundant in all of the above groups. This species is described in the literature as shoaling, usually close to the bottom but sometimes pelagic and close to the surface. Because of its consistent occurrence in the demersal trawl it is included in this analysis. However, it is not clear whether, and in what way, the massive presence of the species on the bottom, especially during daytime, affects the more typically 'demersal' assemblages and whether it is trophically related.

\section{Group 4 - Subthermocline sparid assemblage, from northern Angola to Benguela}

This group includes 45 stations at an average depth of about $87 \mathrm{~m}$, temperature of $18^{\circ} \mathrm{C}$, salinity $35.7 \%$, oxygen concentration $2.4 \mathrm{ml} \mathrm{l}^{-1}$. The grab samples taken in these areas show that the bottom mainly consists of sand, varying from coarse to fine. Several seabream species (family Sparidae) dominate this assemblage that broadly coincides with Longhurst's 'subthermocline sparid subcommunity' (1965) of the Gulf of Guinea, also described for Congo by Durand (1967) and by Fontana (1981). The semi-pelagic Trachurus trecae dominates the catches both in biomass and numbers (28 and 38\%, respectively; Table 5) and was present at $80 \%$ of the stations. Pagellus bellottii and the Angola dentex Dentex angolensis also display a high frequency of occurrence but they represent only 9 and $3 \%$ of the catches respectively. The Congo dentex $D$. congoensis, the bogue Boops boops, and the gurnards Lepidotrigla carolae and Chelidonichthys gabonensis are distributed mostly north of $9^{\circ} \mathrm{S}$ with the pink and large-eye dentex $D$. gibbosus and D. macrophthalmus in the south. It should be noted that $D$. congoensis is a typical tropical representative of the genus while D. macrophthalmus prefers temperate waters and has a typical antitropical distribution, i.e. found on either side of the Equator but with a wide gap in their distribution usually coinciding with the tropical region. Also, the latter is often observed in mid-waters which probably makes it more adapted to avoiding cold and low-oxygen, upwelled waters invading the shelf bottom. Although caught only at the southern stations, this species represents $17 \%$ of the catches (Table 5). Dentex barnardi, another member of this assemblage, is endemic to Angola and Gabon.

\section{Group 5 - Subthermocline assemblage of soft bottoms}

Although no samples of the bottom are available for the areas where this type of assemblage is found, its species composition is indicative of the presence of soft bottoms. The group consists of 29 stations north of the Congo River as well as southward, a little deeper than stations of Group 4, with depth between 70 and $140 \mathrm{~m}$, average temperature of $17^{\circ} \mathrm{C}$, high salinity $(35.7 \%$ ) and oxygen levels of $2.2 \mathrm{ml} \mathrm{l}^{-1}$ These stations were quite distinct in species association as compared to those described under Group 4, at a similar depth range. Fifty-two per cent of the total catches within this group consists of the splitfin Synagrops microlepis, a species mainly of the upper slope. Trichiurus lepturus, indicative of soft substrate, and Trachurus trecae and Dentex angolensis, known to occur on various types of bottom, are the other dominating species. The blackmouth croaker Pentheroscion mbizi also characterizes the area north of the Congo River, substituted by the splitfin S. microlepis in the more southward stations of this group. P. mbizi was described by Longhurst (1962) as an important species in the subthermocline sparid community in the Gulf of Guinea, and is not part of the most typical sparid community described in Group 4. Other species typifying this group are: the lizardfish Saurida brasiliensis, the bearded brotula Brotula barbata, the longfin bonefish Pterothrissus belloci, the shortfin squid lllex coindetii and the deepwater rose shrimp Parapenaeus longirostris.

\section{Group 6 - Subthermocline assemblage between Tombua and Cunene}

This region is characterized by the lack of a sharp, inshore thermocline because of almost continuous upwelling. The term 'subthermocline' should perhaps be abandoned here and used only for the northern region, widely influenced by a tropical structure of the water masses. This group includes 24 stations at an average depth of $88 \mathrm{~m}$, temperature of $16{ }^{\circ} \mathrm{C}$, salinity $35.3 \%$ (possibly South Atlantic Central Water) and low oxygen levels (average $1.8 \mathrm{ml} \mathrm{l}^{-1}$ ), well below those found at similar depths in the northern regions of the Angolan coast. Species composition greatly differs from that found in the northern regions, as could be expected from the dramatic changes in the hydrological regime. Dominating species are Dentex macrophthalmus (39\% in biomass; Table 5) and the Cape horse mackerel Trachurus capensis (32\%), but other species like $T$. trecae, the African weakfish Atractoscion aequidens, the European squid Loligo vulgaris, the wedge sole Dicologoglossa cuneata and the John dory Zeus faber are also important elements of this assemblage. Pterothrissus belloci and Synagrops microlepis occurred in the deepest stations but considerably shallower than in northern Angola. An interesting feature of a number of species found in this area is that they either have an antitropical distribution, or, although found throughout the tropical region, they are most 
abundant north and south of it where they occur in shallower waters. Dentex macrophthalmus is an example of the first category. It is known to occur along the West African coast from the Strait of Gibraltar to Cape Verde and from Congo to Namibia but it is most abundant off Morocco and southern Angola, i.e. in the colder subtropical regimes. Dicologoglossa cuneata is very abundant on the Moroccan shelf at intermediate and shallow waters and becomes abundant again in southern Angola. It is known to occur at greater depths $(400 \mathrm{~m})$ off Mauritania. It was not reported from the Gulf of Guinea by the Guinean Trawling Surveys (1963/1964). This phenomenon shows the affinity of the above species for colder waters, their appearance on the intermediate shelf being made possible by the occurrence of colder upwelled water.

The upper slope was not sampled throughout and stations are available from north of the Congo River, and from about Ambriz to Benguela. Two main groups are identified (Groups 7 and 8): from about 200 to $350 \mathrm{~m}$ depth and stations deeper than that. The main distinction in species composition between the 2 groups is that in the first there are still a number of shelf species not found in the deeper stations where, on the other hand, more typically slope species appear.

\section{Group 7 - Upper edge of the continental slope}

Fifteen stations are included in this group, at an average depth of $256 \mathrm{~m}$, temperature of $12{ }^{\circ} \mathrm{C}$, salinity of $35.3 \%$ and oxygen levels of $1.2 \mathrm{ml} \mathrm{l}^{-1}$. Synagrops microlepis makes up $52 \%$ in biomass, $66 \%$ in numbers and is found at all stations of this group. The 2, more typically upper slope dwellers, the Atlantic green-eye Chlorophthalmus atlanticus and the Benguela hake Merluccius polli, are the next most abundant and frequent species while Pterothrissus belloci, Parapenaeus longirostris and Illex coindetii show a high frequency of occurrence but lower abundance.

\section{Group 8 - Deeper continental slope}

Seventeen stations were sampled, with a wide range of depths (most between 350 and $550 \mathrm{~m}$, one station at $750 \mathrm{~m}$ ). Temperature was $7.9^{\circ} \mathrm{C}$, salinity $34.8 \%$ and oxygen $1 \mathrm{ml} \mathrm{l}^{-1}$. Most of the stations were sampled during nighttime, when many of the benthopelagic slope species migrate toward the surface. However, a number of typically slope species appear at these stations: Merluccius polli, the dominant species (about $40 \%$ of the biomass), several deep-water shrimp species like the African spider shrimp Nematocarcinus africanus (32\% of the catches; Table 5), the scarlet shrimp Plesiopenaeus edwardsianus and the striped red shrimp Aristeus varidens, the former being the most abundant. Codlings of the genus Laemonena, Benthodesmus thenuis and members of the family Macrouridae, also typify this slope area. Centrophorus granulosus is a large (to $150 \mathrm{~cm}$ ) deep-water shark of the continental slope. It is known to feed on hake and myctophids.

\section{DISCUSSION}

\section{Species assemblages}

Depth is often the main gradient along which faunal changes occur when analysing shelf and upper slope assemblages (Fager \& Longhurst 1968, Lleonart \& Roel 1984, McManus 1985, Roel 1987, Bianchi 1991). The plot of all stations on DCA Axes 1 and 2 (Fig. 6) clearly shows how the station groups are arranged from left to right according to increasing depth, i.e. from the shallow water group to the deepest slope stations. Axis 1 is in fact highly correlated with depth (Table 2, Fig 10). In the present study temperature showed an even greater correlation with DCA Axis 1 (Table 2, Fig. 10) and some of the main groupings of the shelf stations are clearly related to thermal stratification (Fig. 5, Table 4). For this reason the terms supra-, intra- and subthermocline are used in this study to designate the major subdivisions of the shelf stations. The importance of the presence of a sharp thermocline in the distribution of demersal fish was already shown for the Sierra Leone shelf and successively for the whole Gulf of Guinea (Longhurst 1958, 1969 respectively). The northern and central Angolan shelves seem to belong to that regime while the southern part, characterized by almost permanent upwelling, diverges from that pattern. However, while in large areas of the Gulf of Guinea this structure is permanent, off Angola the thermocline may be disrupted by the occurrence of seasonal upwelling and differences in species diversity in shallow waters should be expected. There is no clear, strong oxycline but the high correlation between oxygen and DCA Axis 1 probably accounts for the differences in oxygen concentrations found in the shelf stations (Fig. 10). The 2 intra-thermocline assemblages (Groups 2 and 3) and the 3 subthermocline shelf assemblages (Groups 4 to 6) overlap strongly along Axis 1 (Fig. 6). Species composition in these groups indicates that bottom type may play an important role in the configuration of these assemblages. A further analysis of the subthermocline shelf stations, between 

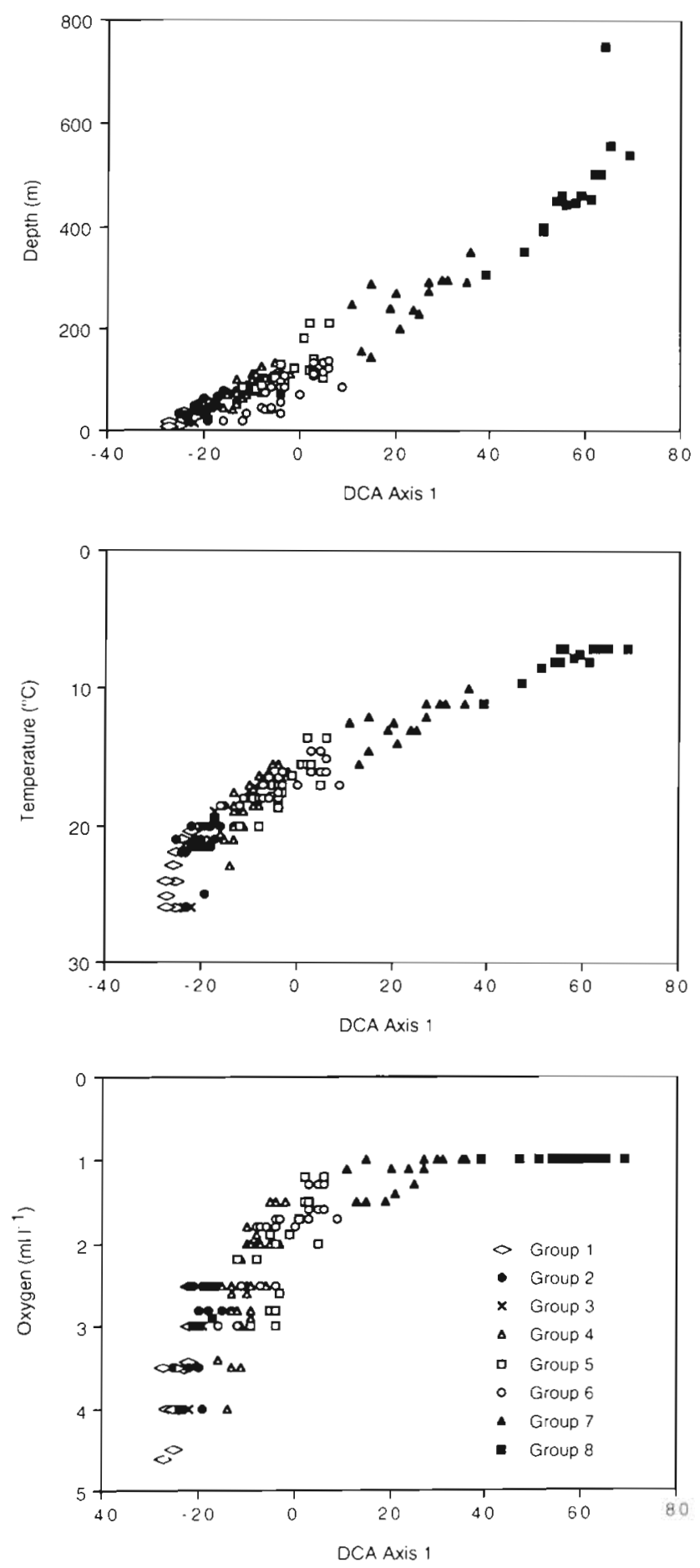

Fig. 10. Plots of DCA Axis 1 against depth, temperature and oxygen

about 70 and $150 \mathrm{~m}$ depth, (Fig. 7) shows that latitude is highly correlated with Axis $1(r=0.86)$. Correlations with temperature, salinity and oxygen are weaker, but still significant (Table 3). This reflects the clear separation between the assemblage found in the south, corresponding to the northern limit of the Benguela Current, and the 2 found north of Benguela which more resemble the typical assemblages originating from the Gulf of Guinea. The other 2 subthermocline shelf assemblages are well separated along Axis 2 which shows some correlation with depth only. Their separation, as can be judged from the species composition, appears to be related to bottom type. The above results agree with those of Domain (1980) for the Mauritania-Senegal shelf where in a similar way Axis 1 of Correspondence Analysis was related to depth and thermal stratification and Axis 2 to bottom type.

\section{Biomass}

Biomass densities were calculated by depth strata and for 3 major species groups, classified according to their depth-distribution range: suprathermocline species, those never below the lower limit of the thermocline (approximately $50 \mathrm{~m}$ depth), including mostly species of the shallow-water Group 1; eurybathic, those species found well above and below the thermocline from shallow, inshore waters to $100 \mathrm{~m}$ depth), typically represented by Brachydeuterus auritus, Trichiurus lepturus, Pagellus bellottii and Balistes capriscus; and finally the subthermocline species, never found in shallow waters and usually below the thermocline. This classification is obviously valid for the northern and central parts of the Angolan shelf while for the southern part, where the thermocline does not meet the shelf, the classification adopted separated only shallow-water and deepwater species.

The northern part of the area (from Cabinda to Luanda) shows the interesting feature, already observed in Congo by Fontana (1981), that the eurybathic species reach their highest biomass densities where the thermocline meets the shelf, while this zone clearly represents a point of separation between the suprathermocline and subthermocline groups (Fig. 9). In deeper waters densities decrease and reach a minimum between 50 and $100 \mathrm{~m}$ depth. At the shelf-break/ upper slope region the highest bottom-trawl catches were obtained consisting mainly of Synagrops microlepis. This species is benthopelagic, migrating to upper water layers at night. It probably feeds on small mesopelagic fishes as deduced from the mouth anatomy: superior and with conical long teeth. Domain (1980) also reports this species as very abundant at the level of the upper slope off Senegal-Gambia and suggests its potential economic value. In deeper waters, Merluccius polli and Nematocarcinus africanus are the most abundant species 
The intermediate region, between Pta. das Palmeirinhas and Benguela, shows a similar pattern to the one described above but with higher values of mean biomass densities for both the suprathermocline and eurybathic species. Brachydeuterus auritus is also very abundant in shallow waters and to $100 \mathrm{~m}$ depth. This species is probably one of the most plentiful fishes of shallow and intermediate waters of West Africa, from Senegal to northern and central Angola (Raitt \& Sagua 1969). The success of this species might be related to its capability of adapting to different water temperatures. This feature must be important especially in the areas with seasonal upwelling as is the case for northern and Central Angola as well as several coastal areas of the Gulf of Guinea. Balistes capriscus has received much attention because of its tremendous increase in biomass in the Gulf of Guinea since the early 1970's, possibly a consequence of overfishing of Sardinella aurita, and its sudden decline in biomass in later years. Though basically demersal (a reef-fish genus) this species also moves to mid-waters to feed on plankton. In Angola it was encountered only in the central part of the country, where the ecological conditions are quite similar to those found off Ghana, i.e. strong seasonal upwelling. No such increase in biomass has, however, occurred off Angola. A second and highest peak in biomass densities is found between 200 and $300 \mathrm{~m}$, consisting mainly of Chlorophthalmus atlanticus and, to a lesser extent, Synagrops microlepis and Merluccius polli.

There is a different situation in the area between Tombua and Cunene. The eurybathic species described above are very rare and the shelf is dominated by Dentex macrophthalmus. It should be noted that the shelf is very steep in its shallowest part and bottom trawl stations are available from about $70 \mathrm{~m}$ depth. Furthermore the shelf edge and upper slope are also very steep and, therefore, no data are available for this region. Biomass densities of the 50 to $100 \mathrm{~m}$ depth stratum are highest in this area as compared with the 2 areas above, where this depth stratum coincides with a minimum biomass (Fig. 9)

A comparison of northern and central Angola areas with the region between southern Mexico and Nicaragua (Bianchi 1991), also subject to seasonal upwelling, shows a similar distribution in the demersal biomass, with highest concentrations on the deeper shelf and upper slope areas and a minimum approximately between 50 and $100 \mathrm{~m}$ depth. The total biomass densities by depth stratum are much higher in the Eastern Central Pacific. However, little fishing occurs in the intermediate and deep waters of that region while Angolan waters have been subject to high fishing pressure for at least $20 \mathrm{yr}$.

\section{Faunal changes with latitude on the shelf area}

The analysis of faunal changes with latitude has necessarily to be performed according to depth strata. A meaningful stratification seems to be: suprathermocline, shallow water assemblages and intermediate shelf assemblages. The upper slope is not included because of lack of adequate sampling especially in the southern part of the area.

As for the suprathermocline species, the tropical type of assemblage follows the inshore, warm equatorial waters which in summertime are transported by a southward flow to Lobito-Benguela and, at times, to Baia dos Tigres. Although some tropical species are found here (e.g. Sardinella maderensis and Pomadasys incisus) the more typical tropical assemblage is usually not found south of Lobito.

With respect to the intermediate-shelf, subthermocline assemblages, a major faunal change occurs in the southern part of the area. As already mentioned, the species associations found between Tombua and Cunene differ greatly from those found in northern and central Angola and the narrow shelf between Benguela and Tombua is where the major turnover of species occurs. The Angola-Benguela frontal system characterizes the area between Tombua and Cunene, and south of Baia dos Tigres upwelling is constant throughout the year. It is therefore not surprising that the fauna typifying this region is different from the one found in the north. Also, the extremely narrow shelf between Benguela and Tombua might function as a physical barrier to the spreading of 'northern' species to the south and vice versa.

Several authors have discussed the position of the zoogeographic boundary separating the tropical Eastern African Region from the temperate South African Region (Briggs 1974) and suggested, on the basis of the distribution of different vertebrate and invertebrate groups, various latitudes ranging from $14^{\circ} \mathrm{S}$ to $18^{\circ} 30^{\prime} \mathrm{S}$. In particular, Longhurst (1962) discussed the distribution of the demersal fish fauna and concluded that the oceanographic frontal zone at about $14^{\circ} \mathrm{S}$ formed a very important boundary. The present study confirms the view that the frontal zone constitutes a major faunal boundary. However, it should be emphasized that this boundary is obviously not a stable one and a latitudinal displacement of species should be expected in connection with the seasonal and the possible inter-annual fluctuations of the front.

Acknowledgements. I thank Tore Høisæter, Gunnar Sætersdal and 3 anonymous reviewers for reading the paper and providing valuable suggestions for its improvement, and Stále Kolbeinson for drawing the figures. 
Appendix 1. Two-way station by species table resulting from the program TWINSPAN. Values denote categories of abundance: 1 : $W<10 \mathrm{~kg} ; 2: 10<W<100 \mathrm{~kg} ; 3: 100<W<1000$ $\mathrm{kg} ; 4: 1000<W<10000 \mathrm{~kg} ; 5: W>10000 \mathrm{~kg}$. Station groups are given along top margin
Penceus notialis Sardinella maderensis Stromateus fiatola

Pseudotolithus seneqalenisis

Nisha africana

Pteroscion pelis

Selene dorsalis

Galeoides decadactylus

Sphyraend guachancho

Chloroscombus chrysurus

Trichiurus leplurus

Brachydeuterus auntus

Scomberomorus tritor

Sparus caeruleostictus

Balistes capriscus

Engraulis encrasicolus

Pomadasys jubelini

Decapterus rhonchus

Lagocephalus leevigatus

Pomadasys incisus

Sphyraena sphyraena

Sardinella aurita

Brotula barbita

Alloteuthis africana

Epunephelus aeneus

Lithograthus mormyrus

Dentex canariensis

Chaetodon hoelers

Fistularia petimba

Dentex barnardi

Pagellus bellottii

Raja miraletus

Sparus pagrus africanus

Saurida brasiliensis

Citharus inguatula

Dentex angolensis

Lepidotrigla cadmani

Dentex gibbosus

Pentheroscion mbizi

Squatina oculata

Torpedo torpedo

Boops boops

Dentex congoensis

Lepidotrigla carolese

Trachurus trecae

Spicara alta

Umbrina caneriensis

Chelidonichlinys gabonerisis

Sepia officinalis

Trachinus armatus

Trachurus capensis

Mustelus mustelus

Atractoscion aequidens

Lepidopus caudatus

Anthias anthias

Loligo vulgaris

Dentex macrophthaimus

Dicologogiossa cuneata

Zeus faber

Triglalyra

Merluccius capensis

Hex conndeti

Synagrops microlepis

Parapenaeus longurostris

Todaropsis eblanae

Pterothrissus belloct

Pontinus spp.

Zenopsis conchifer

Solenocera africana

Chlorophthalmus atianticus

Myctophidaé

Galatheidae

Coelorinchus coelorhincus

Hymenocephalus spp

Laemonaema spp

Penstedion cataphractum

Maldcocephalus occidaembals

Nezurwia aequalis

Merluccius pollt

Etnopterus spp.

Dibranchus atlanticus

Physiculus spp

Polychaeidae

Aristeus varidens

Plesiopenceus edwardsianus

Benthodestius Spp

Nematocarcinus africanus

Centrophorus granulosus

Monomitopus spp

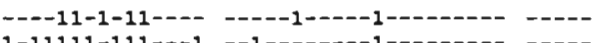

1-11111-111---1 --1--------1-------- ----

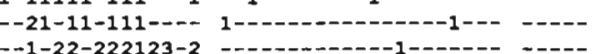

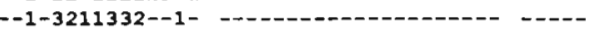

--2-22-2221--- - -

213122122112322 -22-11122-122-1111-1- 1--1-

$--2-22--1212312$-11--1-------1---121- 1----

1-1-212--223312 1-2-21111--2-12----11 -121-

2233212-221-221 11--1-1111-----1111-- -----

- -3322-2211--11 $1-2-2-1122223-2133323$ - 1--

223-2213222-322 ---1-2--1212423233321 ---21

12--1-1---11--2 -----1-11----1----- -11--

----------221- $2133222-0 .-0--2221-\ldots$

---------311 --1-1--11-12-1-11111- - -

$--2-2---12--2-$-11-----1--321----1-- 1--1-

22-.-....-- --1--11-1--1------- 1--11

$11 \cdots \ldots \ldots-11-111111-1-1----1-1-1$

-----1-----3--- -1-----c--3--- 32221 3----

$-------1--131-----1---2-11-111-----1-11$

$1111---0----1$--1--1---1----1--- --11

-

[............ -

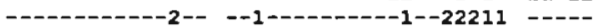

- -

-

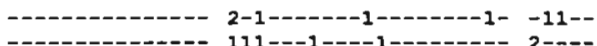

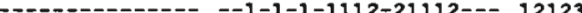

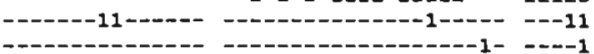

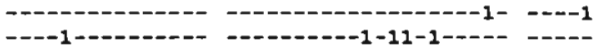

-

-

-

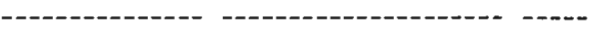

-

-

-

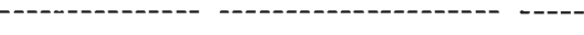

$--1---1----1-1-111----11-2121-1-1-----$

-

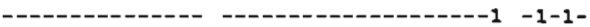

-

$--2-0-2-0-1$

-

-

- - - - - - - - - - - - - - - -

-

$--1$

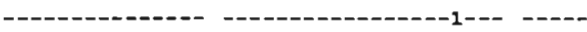

- - - - -

-

- - - -

-

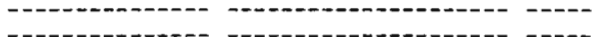

(1)

-

-

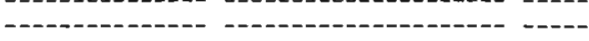

-

-

-

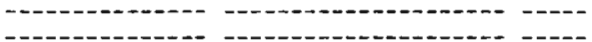

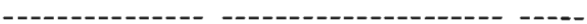

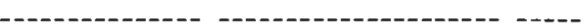

-

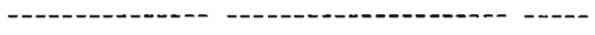

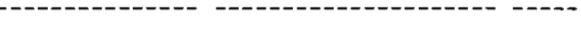

- -

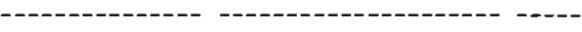

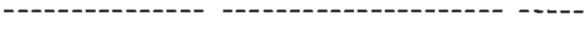

- -

-

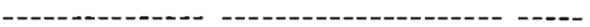

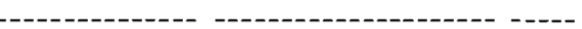

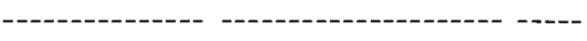

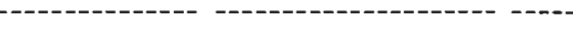




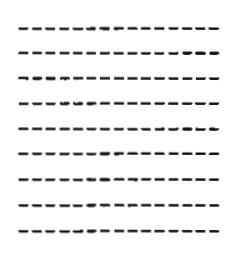

\section{2}
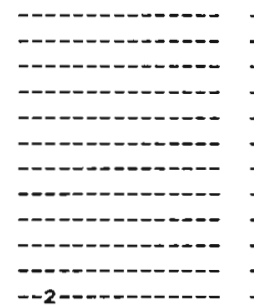

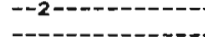

-
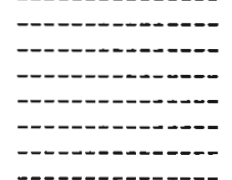

1-0-1-

\section{1-2--}

-

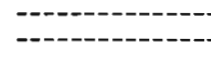

-

-

-

-

-

-

-

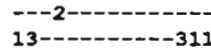

13----------311 $1--1-1-$ ---------2333433334333222 212121221111-11 $112-11222--2212$ 11---------111----111----1-1$-3-321-23322233$ ---121--1-1-1$---111-----11$ $-21-------11$ -------11-1---1-1---------2--2 212-22232122233 - - $1-----11$ -----1-11--------1---.--. -

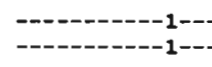
-

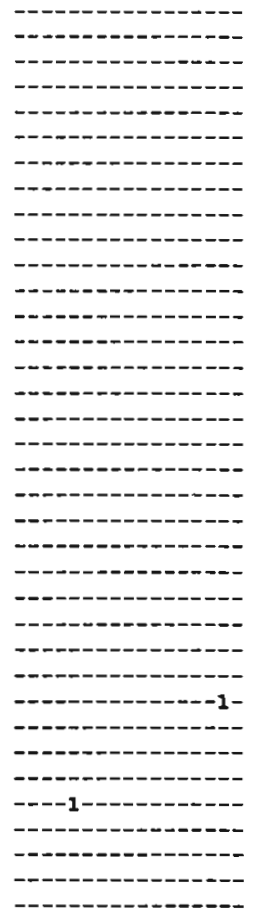
--_------------------------- -

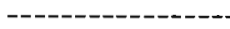

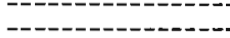
--o-

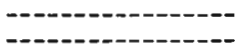
$---1---122-2-21$ 11--1-11-----11$11--1-11--$ -----.---11--. $---2212---$ -------1-.--$---1111------$ 1-1-11-----1--1 -11-11-----------1112-2-----$-22212222111----1$ --1-1111-------1--11-11--1-----1 $-2222233132322232$ --11---2-1---1--1 111111121--2-11111-111

---1-1--1---11111 1121--1-111-11111 1111---1112--i-1 --111211-2-21-1-2 13--222221342-223 $--222-2-\cdots-2-22$ 


\section{LITERATURE CITED}

Berrit, G. R. (1976). Les eaux froides côtières du Gabon à l'Angola sont-elles dues à un upwelling d'Ekman? Cah. ORSTOM, sér. Océanogr. 14: 273-278

Bianchi, G. (1986). Fichas FAO de Identificação de espécies para propósitos comerciais. Guia de campo para as espécies comerciais marinhas e de aguas salobras de Angola. Preparado com o apoio da NORAD e da FAO (FIRM) Programa Regular. FAO, Roma

Bianchi, G. (1991). Demersal assemblages of the continental shelf and slope edge between the Gulf of Tehuantepec (Mexico) and the Gulf of Papagayo (Costa Rica). Mar. Ecol. Prog. Ser. 73: 121-140

Briggs, J C. (1974). Marine zoogeography. McGraw-Hill Book Company, New York

Da Franca, P. (1968). Breves comentários àcerca da biogeografia marinha angolana. Notas Cent. Biol. aquat. Trop. (Lisbon) 12: 1-22

Dias, C. A. (1983). Preliminary report on the physical oceanography off southern Angola, March and July 1971. ICSEAF, 1983 (Part II). Colln. scient. Pap. Int. Commn SE. Atl. Fish. 103-116

Domain, F. (1972). Poissons démersaux du plateau continental sénégambien. Application de l'analyse en composantes principales à l'étude d'une série de chalutages. Cah. ORSTOM, sér. Océanogr. 10: 111-123

Domain, F. (1980). Contribution à la connaissance de l'écologie des poissons démersaux du plateau continentale sénégalo-mauritanien. Les ressources démersales dans le contexte général du Golfe de Guinée. Thèse de Doctorat d'Etat es - Sciences Naturelles. Université Pierre et Marie Curie, Paris VI

Durand, J.-R. (1967). Etude des poissons benthiques du plateau continental congolais. 3e partie: étude de la repartition, de l'abondance et des variations saisonnières. Cah. ORSTOM, sér. Océanogr. 5: 3-68

Fager, E. W. (1957). Determination and analysis of recurrent groups. Ecology 38(4): 586-595

Fager, E. W., Longhurst, A.R. (1968). Recurrent group analysis of species assemblages of demersal fish in the Gulf of Guinea. J. Fish. Res. Bd Can. 25: 1405-1421

Fischer, W., Bianchi, G., Scott, W. B. (eds.) (1981). FAO species identification sheets for fishery purposes. Eastern Central Atlantic; fishing areas 34, 47 (in part). Canada Funds-in-trust. Ottawa, Department of Fisheries and Oceans Canada by arrangement with the Food and Agriculture Organization of the United Nations, Vols. 1-7

Fontana, A. (1981). Milieu marin et ressources halieutiques de la Republique populaire du Congo. Trav. et Doc. ORSTOM 138: 153-211

Green, R. H., Vascotto, G. L. (1978). A method for the analysis of environmental factors controlling patterns of species composition in aquatic communities. Wat. Res. 12: 583-590

Hill, M. O. (1979). TWINSPAN - a FORTRAN program for arranging multivariate data in an ordered two-way table by classification of individuals and attributes. Cornell University, Ithaca, New York

Hill, M. O, Gauch, H. G. (1980). Detrended correspondence analysis, an improved ordination technique. Vegetatio 42 . $47-58$

This article was submitted to the editor
Lleonart, J., Roel, B. A. (1984). Análisis de las comunidades de peces y crustáceos demersales de la costa de Namibia (Atlántico Sudoriental). Investigación pesq. 48: 187-206

Longhurst, A. R. (1958). An ecological survey of the West African marine benthos. Colonial office, Fishery Publ, no. 11

Longhurst, A. R. (1962). A review of the oceanography of the Gulf of Guinea. Bull. Inst. fond. Afr. noire 24:633-663

Longhurst, A. R. (1965). A survey of the fish resources of the Eastern Gulf of Guinea. J. Cons. Int. Explor. Mer 34: $300-334$

Longhurst, A. R. (1969). Species assemblages in tropical demersal fisheries. Proceedings of the symposium on the oceanography and fishery resources of the Tropical Atlantic. Review papers and contributions. (Abidjan, Ivory Coast, 20-28 Oct. 1966). Unesco, Paris

Longhurst, A. R., Pauly, D. (1987). Ecology of tropical oceans. Academic Press, New York

Mas-Riera, J. Lombarte, A., Gordoa, A., Macpherson, E. (1990). Influence of the Benguela upwelling on the structure of the demersal fish populations off Namibia. Mar. Biol. 104: 175-182

McManus, J. W. (1985). Descriptive community dynamics: background and an application to tropical fisheries management. Ph.D. thesis, University of Rhode Island, Providence

Raitt, D. F. S., Sagua, V. O. (1969). Preliminary investigations on the biology of Brachydeuterus auritus (Val. 1831), in Nigerian waters. Proceedings of the symposium on the oceanography and fisheries resources of the tropical Atlantic (Abidjan, Ivory Coast, 20-28 Oct. 1966). Unesco, Paris, France

Roel, B. A. (1987). Demersal communities of the west coast of South Africa. In: Payne, A. I. L., Gulland, J, A., Brink, K. H. (eds.) The Benguela and comparable ecosystems. S. Afr. J. mar. Sci. 5: 575-584

Shannon, L. V., Agenbag, J. J., Buys, M. E. L. (1987). Largeand mesoscale features of the Angola-Benguela front. In: Payne, A. I. L., Gulland. J. A., Brink, K. H. (eds.) The Benguela and comparable ecosystems. S. Afr. J. mar. Sci. 5: $11-34$

Stromme, T., Sæetersdal, G. (1991). Surveys of the fish resources of Angola, 1985-86 and 1989. Reports on surveys with RV 'Dr. F. Nansen' Institute of Marine Research, Bergen, Norway

ter Braak, C. J F. (1987). CANOCO - a FORTRAN program for canonical community ordination by (partial) (detrended) (canonical) correspondence analysis, principal component analysis and redundancy analysis (version 2.1). ITI-TNO, Groep Landbouwwiskunde, Wageningen

Voituriez, B., Herbland, H. (1982). Comparaison des systèmes productifs de l'Atlantique tropical est: dômes thermiques, upwellings cótiers et upwelling équatorial. Rapp. P.-v. Réun. Cons. int Explor Mer 180: 114-130

Wauthy, B. (1977). Revision des classifications des eaux de surface du Golfe de Guinée (Berrit,1961). Cah. ORSTOM, sér. Océanogr. 15: 279-295

Wauthy, B. (1983). Introduction à la climatologie du Golf de Guinée. Océanogr. Trop. 18: 103-138

Manuscript first received: July 3, 1991

Revised version accepted: February 11, 1992 